\title{
ARTICLE
}

\section{Actin nucleation at the centrosome controls lymphocyte polarity}

Dorian Obino', Francesca Farina², Odile Malbec, Pablo J. Sáez¹, Mathieu Maurin, Jérémie Gaillard², Florent Dingli ${ }^{3}$, Damarys Loew ${ }^{3}$, Alexis Gautreau ${ }^{4}$, Maria-Isabel Yuseff ${ }^{5}$, Laurent Blanchoin ${ }^{2}$, Manuel Théry ${ }^{2,6}$ \& Ana-Maria Lennon-Duménil ${ }^{1}$

Cell polarity is required for the functional specialization of many cell types including lymphocytes. A hallmark of cell polarity is the reorientation of the centrosome that allows repositioning of organelles and vesicles in an asymmetric fashion. The mechanisms underlying centrosome polarization are not fully understood. Here we found that in resting lymphocytes, centrosome-associated Arp2/3 locally nucleates F-actin, which is needed for centrosome tethering to the nucleus via the LINC complex. Upon lymphocyte activation, Arp2/3 is partially depleted from the centrosome as a result of its recruitment to the immune synapse. This leads to a reduction in F-actin nucleation at the centrosome and thereby allows its detachment from the nucleus and polarization to the synapse. Therefore, F-actin nucleation at the centrosome-regulated by the availability of the Arp2/3 complex-determines its capacity to polarize in response to external stimuli.

\footnotetext{
${ }^{1}$ INSERM-U932 Immunité et Cancer, Institut Curie, PSL Research University, 75248 Paris Cedex 05, France. ${ }^{2}$ CytoMorpho Lab, Biosciences \& Biotechnology Institute of Grenoble, UMR5168, CEA/INRA/CNRS/Université Grenoble-Alpes, Grenoble 38054, France. ${ }^{3}$ Laboratoire de Spectrométrie de Masse Protéomique, Institut Curie, PSL Research University, 75248 Paris Cedex 05, France. ${ }^{4}$ Ecole Polytechnique, CNRS UMR7654, Palaiseau 91120, France. ${ }^{5}$ Departamento de Biologia Celular y Molecular, Pontificia Universidad Catolica de Chile, Santiago 6513677, Chile. ${ }^{6}$ CytoMorpho Lab, Hopital Saint Louis, Institut Universitaire d'Hematologie, UMRS1160, CEA/INSERM/Université Paris Diderot, Paris 75010, France. Correspondence and requests for materials should be addressed to

A.-M.L.-D. (email: Ana-Maria.Lennon@curie.fr).
} 
C ell polarity regulates a broad range of biological processes such as cell division, cell fate and cell migration ${ }^{1-3}$. It relies on the organization of the microtubule cytoskeleton, which defines the axis of cell division, as well as the directionality of intracellular trafficking ${ }^{4}$. As the centrosome drives the nucleation and organization of microtubules, this organelle was found to play an essential role in the polarization of a variety of cell types ranging from yeast to specialized cells in multicellular organisms ${ }^{5}$. In lymphocytes, centrosome reorientation to one cell pole was shown to be required for cell migration ${ }^{6}$, asymmetric division ${ }^{1,2,7}$ and immune synapse formation ${ }^{8}$.

The term immune synapse refers to the zone of tight interaction that forms between lymphocytes and antigen-presenting cells towards which the centrosome polarizes9. It is viewed as a signalling platform where both exocytotic and endocytotic events needed for lymphocytes to perform their specific effector function take place ${ }^{10}$. These include the secretion of granules in both cytotoxic lymphocytes and natural killer cells ${ }^{11}$, of cytokine-loaded vesicles in helper $\mathrm{T}$ cells ${ }^{12,13}$ and of hydrolase-containing lysosomes in B cells ${ }^{5,14}$. Hence, centrosome polarization emerges as pivotal in the regulation of immunity, stressing the need to unravel the underlying molecular mechanisms. In that regard, PKC and Cdc42 signalling molecules as well as the microtubule minusend motor Dynein were shown to regulate centrosome repositioning at the synapse of both $\mathrm{B}$ and $\mathrm{T}$ lymphocytes ${ }^{14-20}$. Regarding the actin cytoskeleton, Arp2/3-dependent nucleation of F-actin was shown to be dispensable for centrosome polarization in $\mathrm{T}$ lymphocytes, which rather requires the activity of Formins ${ }^{21}$. In general, whether and how centrosome-intrinsic components regulate its ability to polarize remains unexplored.

In this study, we show that Arp2/3-dependent F-actin nucleation at the centrosome of resting lymphocytes links this organelle to the nucleus. Clearance of centrosomal Arp2/3 upon lymphocyte activation promotes centrosome-nucleus separation and subsequent centrosome polarization to the immune synapse. F-actin nucleation at the centrosome therefore determines the ability of this organelle to polarize to one cell pole.

\section{Results}

Lymphocyte activation modifies the centrosome proteome. We aimed at investigating the role of centrosome-associated proteins in cell polarity by using B lymphocytes as a model. Centrosome polarization in these cells can be triggered by engaging their membrane antigen receptor-the B-cell antigen receptor (BCR) - with surface-tethered ligands coated on latex beads (Fig. 1a), planar surfaces or cells $s^{14,17,22}$. We hypothesized that changes in the composition of centrosome-associated proteins between non-polarized and polarized cells might reveal valuable candidates to be involved in this process. A stable isotope labelling by amino acids in cell culture (SILAC) ${ }^{23}$-based quantitative proteomic approach was therefore developed to identify proteins differentially associated with the centrosome of non-polarized and polarized B cells. For this, B cells were grown in cultures containing lysine labelled with light or heavy carbon isotopes and incubated for 60 min with BCR-ligand ${ }^{+}$or BCR-ligand $^{-}$beads, respectively (Fig. 1b). Cells were lysed, centrosomes were isolated on sucrose gradient and the three main $\gamma$-tubulin-containing fractions were pooled for each sample (Fig. 1c). Resulting pools were mixed $1: 1$ to be separated by SDS-polyacrylamide gel electrophoresis (SDS-PAGE) followed by reverse-phase liquid chromatography and analysed by high-resolution mass spectrometry (LC-MS/MS) (Fig. 1b). This led to the quantification of 1,600 proteins (false discovery rate (FDR) of $1 \%$, number of peptides used $\geq 3$; Fig. 1d) among which 835 were differentially associated with the centrosome of activated lymphocytes (absolute fold change $\geq 10 \%$ and adjusted $P$ value of quantification $\leq 0.05$; Fig. $1 d$, light red). To identify key networks, genome ontology (GO) term enrichment was performed on these 835 proteins. As expected, this analysis highlighted components of the microtubule-organizing centre (enrichment factor: 1.9; $P$ value $=3.56 \times 10^{-05}$ ) and the cytoskeleton (enrichment factor: $1.8 ; P$ value $=2.65 \times 10^{-11}$ ) as two major groups of proteins enriched in centrosome preparations (Supplementary Table 1). More surprisingly, zooming on proteins belonging to the GO term 'Cytoskeleton' showed that while microtubule-related components were either increased or decreased in polarized cells, the majority of actin cytoskeleton components were reduced (69.8\%; Fig. 1e and Supplementary Table 2). Noticeably, this particularly applied to three subunits of the branched actin-nucleating complex Arp $2 / 3$ (ref. 24; 10 and 12\% decrease; Fig. 1e, red; and Supplementary Tables 3-5). Immunoblot analysis showed an even more pronounced reduction of the Arp2/3 subunit Arp2 in centrosomal fractions from activated lymphocytes (Supplementary Fig. 1a). No reduction in the total amount of Arp2 was observed between both conditions (Supplementary Fig. 1b). We conclude that BCR engagement induces multiple changes in the centrosome proteome including a significant reduction in the pool of associated Arp2/3. Although the presence of this complex at the centrosome had been described in the past ${ }^{25}$, whether it regulates centrosome function remains unclear. We therefore focused our analysis on exploring the putative role of Arp $2 / 3$ reduction at the centrosome of activated lymphocytes in the polarization of this organelle.

Reduced centrosomal Arp2/3 in activated lymphocytes. We next asked whether reduction of Arp2/3 at the centrosome was equally observed in intact lymphocytes. Immunofluorescence analysis revealed the presence of two pools of Arp $2 / 3$ in resting B cells: a cortical pool (Fig. 2a, white arrow) and a cytosolic pool that surrounded the centrosome (Fig. 2a, white star). To accurately quantify this centrosome-associated pool of Arp2/3, we computed a radial line scan of Arp2 fluorescence intensity from the centrosome of resting lymphocytes and, based on this result, we defined a 'centrosomal area' (Fig. 2b). The amount of Arp2/3 in this centrosomal area was then quantified at different time points after BCR engagement. In agreement with our proteomic and immunoblot data, we found that this centrosome-associated pool of Arp2/3 gradually decreased in time upon lymphocyte stimulation with BCR-ligand ${ }^{+}$beads (Fig. 2a,c). No reduction in the total amount of Arp2/3 was found (Supplementary Fig. 2a). Similarly, in resting B lymphocytes, we observed the presence of a pool of F-actin in close vicinity of the centrosome (Fig. 2d), which co-localized with Arp2/3 (Supplementary Fig. 2b). In contrast, in lymphocytes incubated for $30 \mathrm{~min}$ with $\mathrm{BCR}$-ligand ${ }^{+}$beads, F-actin was observed as patches dispersed in the cytosol rather than gathered around the centrosome (Fig. 2d). After $60 \mathrm{~min}$ of stimulation, the centrosome polarized to the cell-bead interface and was therefore found in proximity to the cortical F-actin pool. Nonetheless, the pool of centrosome-associated F-actin was decreased in these cells (Fig. 2b,d,e). No reduction in the total amount of F-actin was observed (Supplementary Fig. 2c). Of note, because methanol fixation required for $\gamma$-tubulin staining is not compatible with phalloidin labelling, the centrosome was stained with antibodies directed against $\alpha$-tubulin, and images were processed (fluorescence intensity threshold) to visualize the centrosome but not the microtubules (Supplementary Fig. 2d). Altogether these results show that resting B cells display a pool of Arp2/3 and F-actin at their centrosome that decreases while this organelle polarizes to the immune synapse. 
a

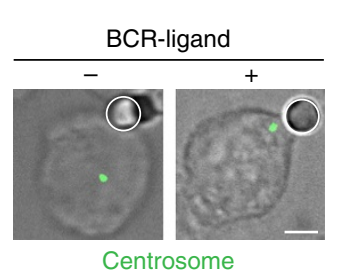

b

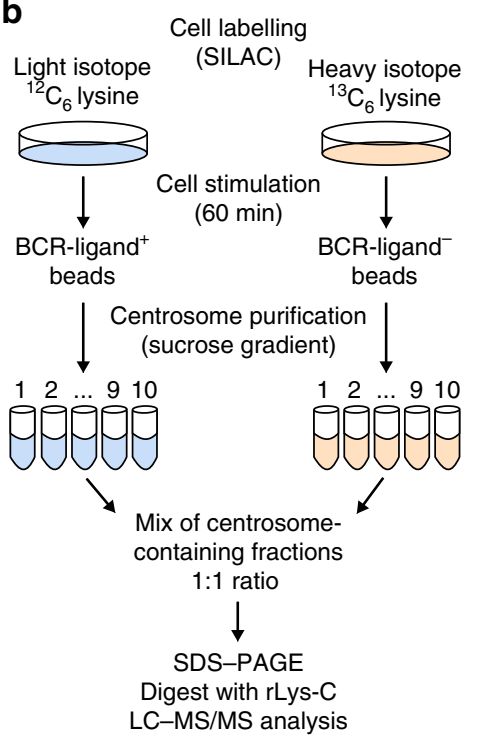

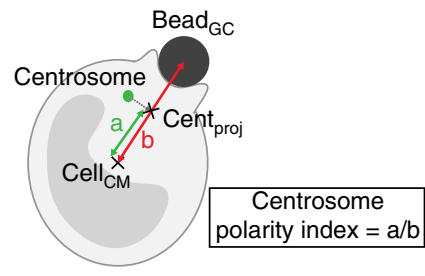

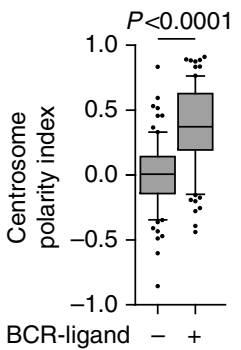

C

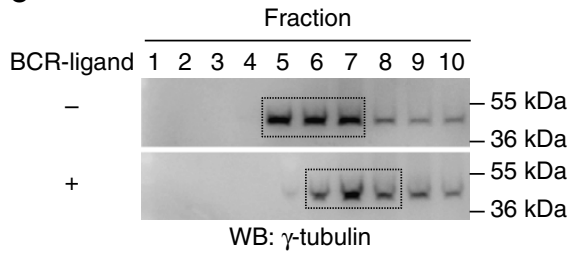

d

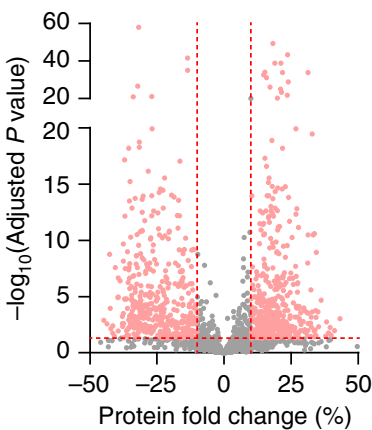

e

Centrosome/microtubule
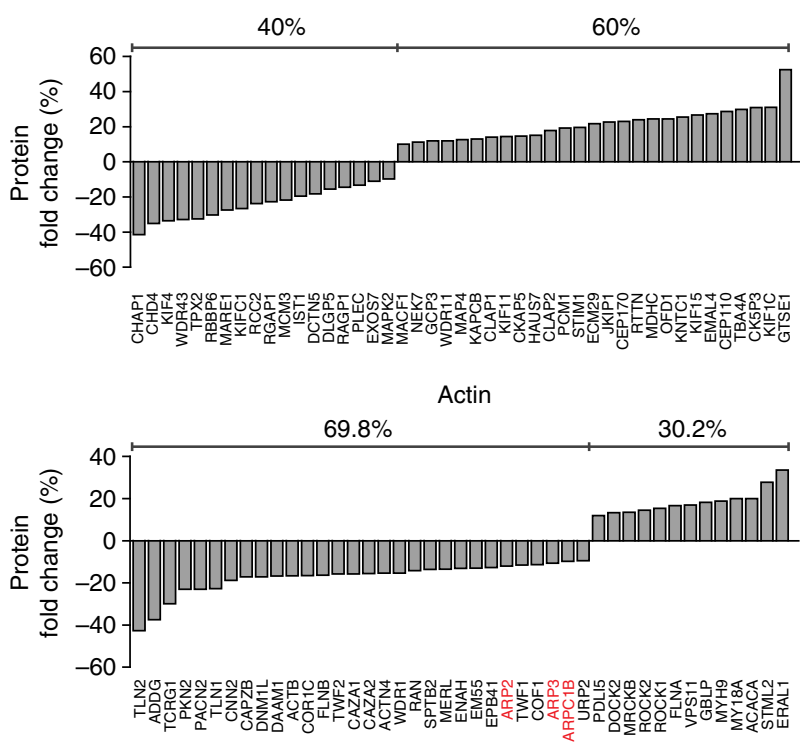

Figure 1 | Lymphocyte stimulation modifies the centrosome-associated proteome. (a) Left: representative images of non-polarized (BCR-ligand ${ }^{-}$) and polarized ( $\mathrm{BCR}$-ligand ${ }^{+}$) $\mathrm{B}$ cells. B cells were incubated for 60 min with beads coated with either BCR ligands or with proteins that do not engage the $B C R$, fixed and the centrosome was stained ( $\gamma$-tubulin). White circles indicate bead position. Scale bar, $3 \mu \mathrm{m}$. Middle: schematics depicting centrosome polarity index measurement. Right: quantification of centrosome polarity index. Data are pooled from three independent experiments with $n=80$ and 85 cells for $\mathrm{BCR}$-ligand ${ }^{-}$and BCR-ligand ${ }^{+}$, respectively. Unpaired Student's $t$-test was used to determine statistical significance. (b) SILAC-based MS workflow used to identify proteins differentially associated with the centrosome of B cells stimulated with either BCR-ligand ${ }^{-}$or $^{B C R}-l i g a n d{ }^{+}$beads. (c) Western blots highlighting centrosome-containing fractions after centrosome isolation on discontinuous sucrose gradient. Immunoblots are representative of three independent experiments. (d) Volcano plot showing the 835 proteins considered for further analysis (light red) among the total of the 1,600 quantified proteins. Horizontal red line represents the threshold for statistical significance (adjusted $P$ value $\leq 0.05$ ). Vertical red lines represent the biological threshold used to select proteins ( $-10 \%$ and $+10 \%$ of protein fold change). (e) Protein fold change (\%) for each of the 45 proteins belonging to the 'centrosome/microtubule' subgroup (top) and the 43 belonging to the 'actin' one (bottom). 
a

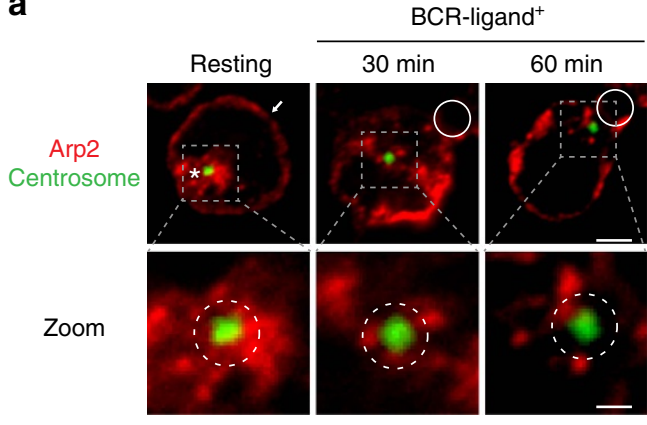

C

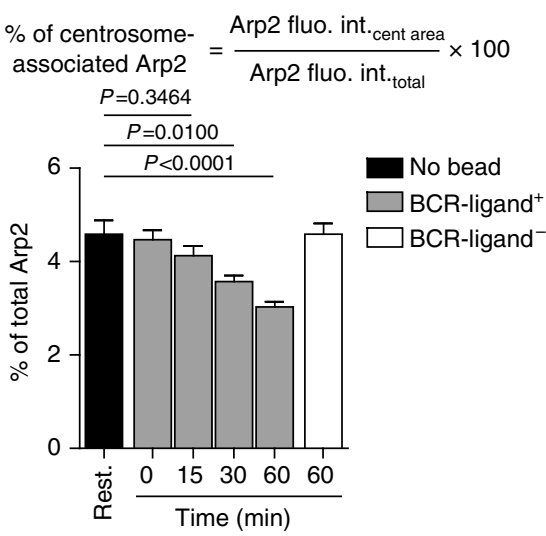

d

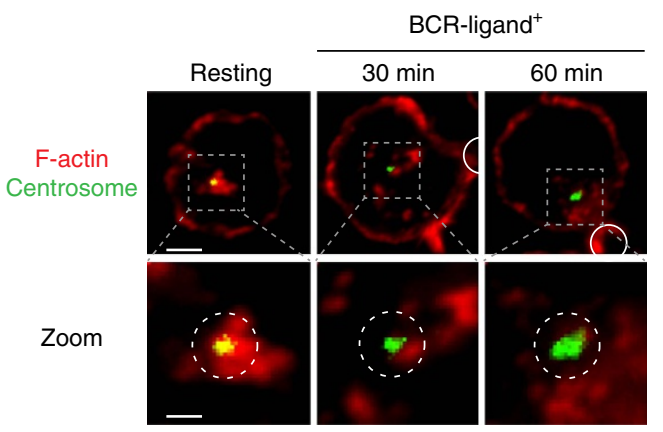

b
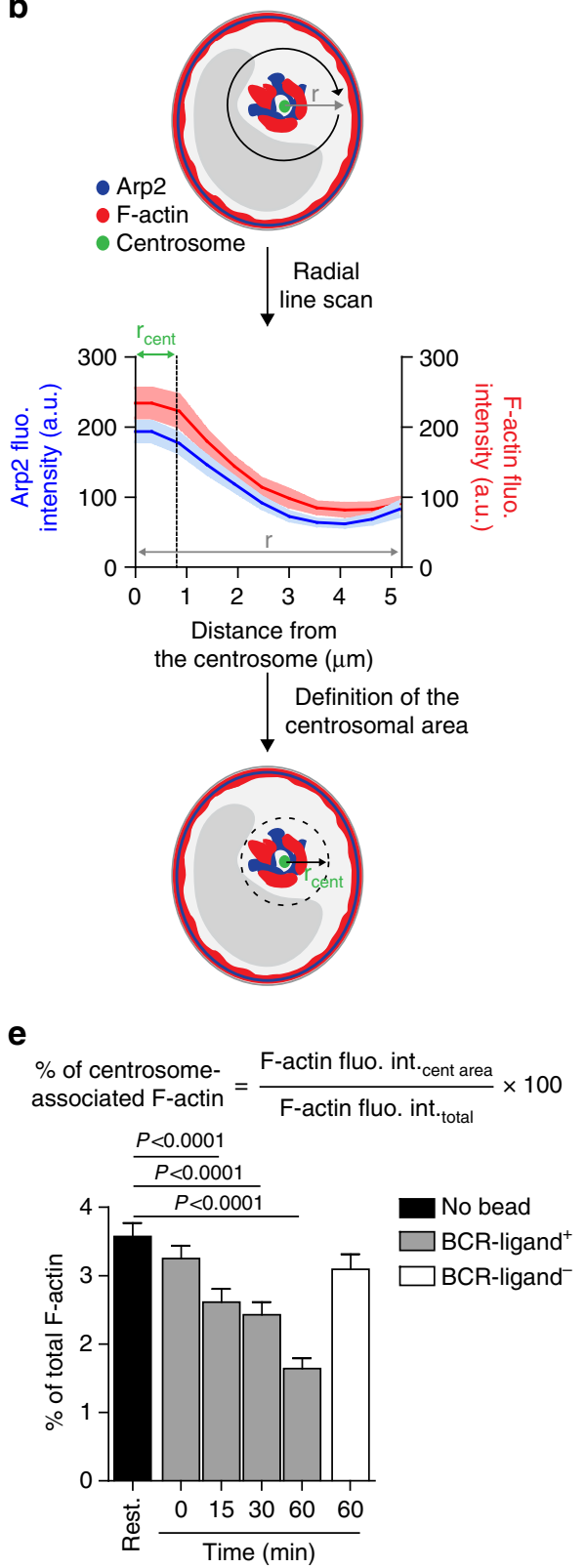

Figure 2 | Decreased association of Arp2/3 and F-actin with centrosomes of BCR-stimulated lymphocytes. (a,d) Representative images of B cells under resting conditions or stimulated with $\mathrm{BCR}$-ligand ${ }^{+}$beads for indicated time, fixed and co-stained for Arp2 (white arrow: cortical pool; ${ }^{*}$ : centrosomal pool) (a) or F-actin (phalloidin) (d) and the centrosome ( $\alpha$-tubulin). White circles indicate bead position. Dashed grey squares indicate the centrosomal region magnified below each image. Dashed circles on bottom panel highlight the centrosomal area used for quantification. Scale bars, top: $3 \mu \mathrm{m}$; bottom: $1 \mu \mathrm{m}$. (b) Schematics depicting the pipeline used to quantify centrosome-associated Arp2 and F-actin. (c,e) Quantification of centrosome-associated Arp2 (c) and F-actin (e) from cells shown in a and d, respectively. Data are pooled from three independent experiments with (c) $n=67,62,64,72,61$ and 69 cells, and (e) $n=54,63,64,62,59$ and 64 cells from left to right, respectively. fluo. int., fluorescence intensity. cent, centrosome. Rest., Resting.

Reduced F-actin nucleation at activated lymphocyte centrosomes. Having recently shown that centrosomes possess an intrinsic actin-nucleating activity in various cell types including $\mathrm{T}$ lymphocytes ${ }^{26}$, we investigated whether the distinct amounts of centrosome-associated F-actin observed in resting and BCRstimulated B lymphocytes reflected different actin nucleation capacities. For this, centrosomes purified from resting and activated B lymphocytes were compared for their ability to nucleate actin filaments in vitro. Strikingly, we observed that both centrosome preparations assembled actin asters from $\gamma$-tubulin spots (Fig. 3a), indicating that centrosomes possess an intrinsic actin nucleation capacity. In agreement with our hypothesis, actin nucleation by centrosomes purified from BCR-stimulated cells was strongly diminished as compared with centrosomes purified from resting lymphocytes. Indeed, both the number of actin asters and the actin fluorescence intensity at the aster centre were significantly decreased when using centrosomes from activated cells (Fig. 3b-d and Supplementary Movie 1). Importantly, centrosome integrity was not affected in preparations from activated lymphocytes, as shown by their ability to nucleate microtubules (Supplementary Fig. 3a). Consistent with these results and with our proteomic and immunofluorescence data, the amount of Arp2/3 associated with centrosomes purified from BCR-stimulated lymphocytes was also found to be strongly 
a

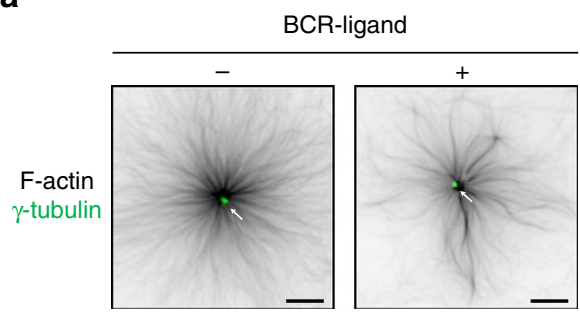

b

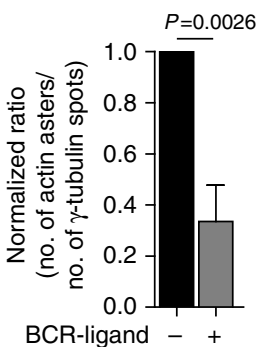

C

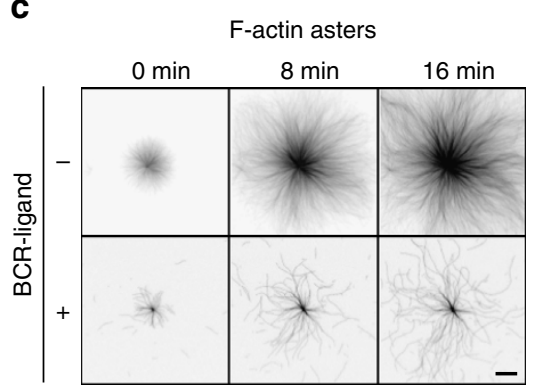

d

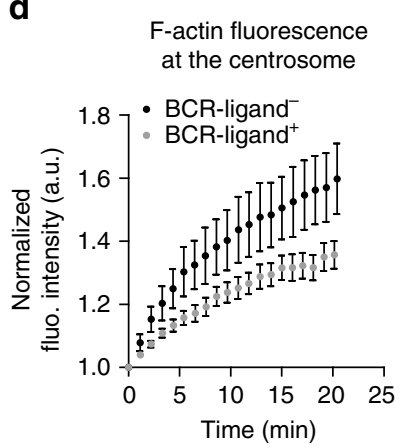

e

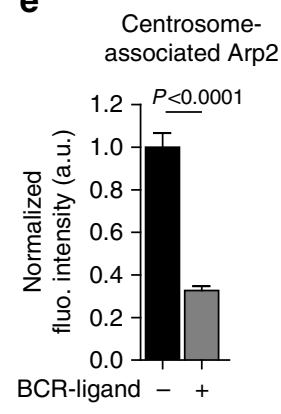

f

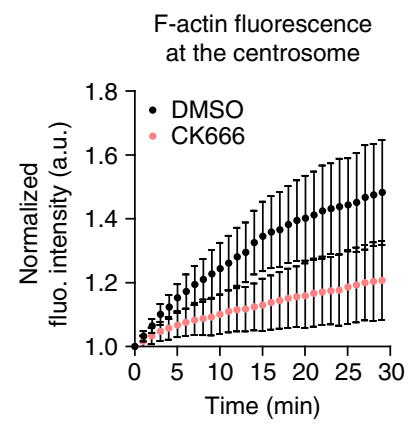

i

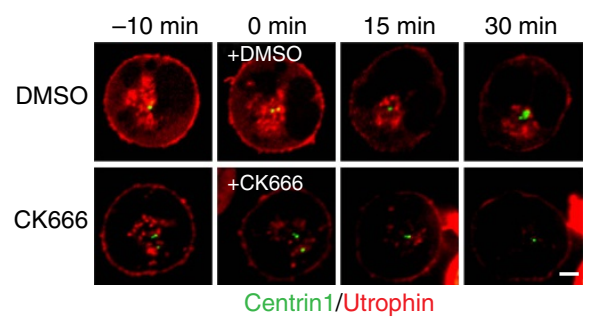

g

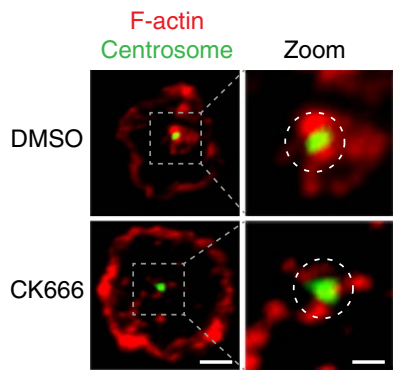

h

Centrosomeassociated F-actin

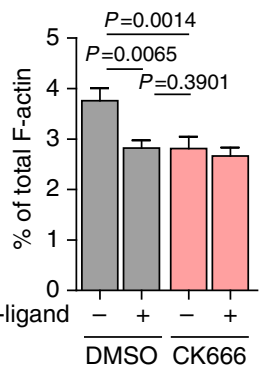

BCR-ligand $\frac{-+}{\mathrm{DMSO}} \frac{+}{\mathrm{CK} 666}$

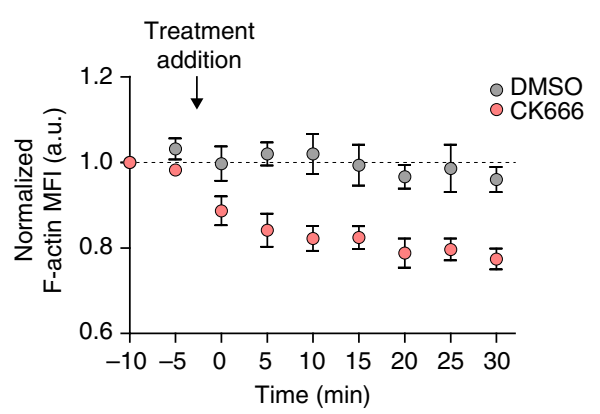

Figure 3 | F-actin nucleation by centrosomes is downregulated upon BCR stimulation. (a) Representative images of actin asters nucleated from isolated centrosomes (white arrow). Scale bar, $8 \mu \mathrm{m}$. (b) Actin nucleation efficiency was calculated as the ratio of the number of actin asters divided by the number of $\gamma$-tubulin spots ( $>200$ actin asters and $>450 \gamma$-tubulin spots per condition pooled from four independent experiments). (c) Sequential images of F-actin assembly by centrosomes isolated from B cells stimulated with indicated beads. Scale bar, $5 \mu$ m. (d) Quantification of F-actin nucleation activity ( $n=14$ and 12 actin asters per condition; data are representative of four independent experiments) (e) Quantification of Arp2 fluorescence (fluo.) intensity associated with purified centrosomes ( $n=100$ and 190 centrosomes per condition pooled from two independent experiments). (f) Quantification of actin nucleation activity of centrosomes purified from resting lymphocytes in the presence of CK666 or dimethylsulfoxide (DMSO; $n=12$ and 22 actin asters, respectively; data are representative of two independent experiments). (g) Representative images of resting B cells treated with DMSO or CK666 for $60 \mathrm{~min}$, fixed and co-stained for F-actin (phalloidin) and the centrosome ( $\alpha$-tubulin). Dashed grey squares indicate the region magnified on the right panel. Dashed circles on the right panel highlight the centrosomal area used for quantification. Scale bars, left: $3 \mu \mathrm{m}$; right: $1 \mu \mathrm{m}$. (h) Quantification of centrosomeassociated F-actin of B cells pretreated for 30 min with DMSO or CK666 before being stimulated with indicated beads for 60 min in presence of the drug ( $>60$ cells per condition pooled from three independent experiments). (i) Left: sequential images of resting B cells co-transfected with the centrosomal marker eGFP-Centrin1 and the F-actin probe Utrophin-RFP imaged by time-lapse spinning disk microscopy and treated with either DMSO or CK666 (between $t_{-5 \mathrm{~min}}$ and $t_{0 \mathrm{~min}}$ ). Scale bar, $3 \mu \mathrm{m}$. Right: quantification of centrosome-associated F-actin over time. Centrosomal F-actin mean fluorescence intensity (MFI) was normalized with respect to initial intensity $\left(t_{-10 \mathrm{~min}}\right)$ for each cell. Data are pooled from two independent experiments and graph shows the mean \pm s.e.m. of $n=8$ and 10 cells for DMSO and CK666, respectively. $P$ values determined by Mann-Whitney test. 
decreased as compared with centrosomes of resting B cells (Fig. 3e).

The involvement of the Arp2/3 complex in F-actin nucleation by centrosomes purified from resting lymphocytes was confirmed by using the CK666 Arp2/3 inhibitor ${ }^{27}$, which significantly reduced F-actin assembly (Fig. $3 \mathrm{f}$ ). On the same line, treatment of resting $\mathrm{B}$ cells with CK666 decreased the amount of centrosome-associated F-actin to the levels observed in BCR-stimulated lymphocytes (Fig. 3g,h). Equivalent results were obtained when silencing Arp2/3 with two different siRNA (Supplementary Fig. 3b,c) or when using the Utrophin-red fluorescent protein (RFP) probe to label F-actin in live-imaging experiments: addition of CK666 resulted in decreased centrosomal F-actin in non-activated lymphocytes (Fig. 3i and Supplementary Movie 2). Interestingly, a significant reduction in the fraction of centrosome-associated Arp2/3 was also observed in CK666-treated resting B cells (Supplementary Fig. 3d), suggesting that Arp2/3 activity is required for its localization at the centrosome and/or that the presence of branched actin at the centrosome might help locally maintaining the complex. Of note, formin inhibition did not decrease F-actin nucleation at the centrosome, indicating that it most likely did not play a direct role in this process. Interestingly, formin inhibition even increased the amount of F-actin at the centrosome, what might result from the recently reported competition between Arp2/3 and formins ${ }^{28}$ (Supplementary Fig. 3e,f). We conclude that lymphocyte centrosomes nucleate F-actin in an Arp2/3-dependent manner and that this property of centrosomes is downregulated upon lymphocyte activation as a result of Arp2/3 local depletion.

Arp2/3 recruitment to the immune synapse. We next searched for the molecular mechanisms responsible for this partial depletion of Arp2/3 from the centrosome of BCR-stimulated lymphocytes. It was shown that the Cortactin homologue haematopoietic lineage cell-specific protein (HS1), which is predominantly expressed in haematopoietic cells ${ }^{29}$, recruits Arp2/3 to the BCR signalosome upon antigenic stimulation ${ }^{30}$. Consistently, we observed that BCR engagement with BCRligand ${ }^{+}$beads induced HS1 phosphorylation and accumulation at the cell-bead interface (Fig. 4a,b). We therefore hypothesized that phospho-HS1-dependent recruitment of Arp2/3 at the immune synapse might lead to its partial depletion from the centrosome and thereby to a local decrease in F-actin nucleation. Consistent with this hypothesis, we found that the gradual decrease in the pool of centrosome-associated Arp2/3 and F-actin was concomitant to the accumulation of both proteins at the cell-bead interface (Fig. 4c-e and Supplementary Fig. 4a). This was also observed for F-actin in time-lapse imaging experiments: upon BCR stimulation, F-actin gradually decreased at the centrosome but progressively increased at the synapse (Fig. 4f and Supplementary Movie 3 and 4). Noticeably, both the decrease of Arp $2 / 3$ and F-actin at the centrosome and their increase at the synapse were severely impaired when silencing HS1 (Fig. 4g,h and Supplementary Fig. 4b). No reduction in centrosome-associated F-actin, nor in the total amount of F-actin was observed between control and HS1-silenced lymphocytes (Supplementary Fig. 4c,d). Hence, HS1-dependent recruitment of Arp $2 / 3$ at the cell-bead interface is associated with its partial depletion from the centrosome, thus decreasing the actin nucleation capacity of this organelle.

Centrosomal Arp2/3 and actin impair centrosome polarization. HS1-silenced lymphocytes that maintained high levels of centrosomal Arp $2 / 3$ and F-actin upon BCR stimulation were next used to investigate whether Arp2/3 and F-actin depletion from the centrosome regulates the ability of this organelle to polarize to the immune synapse. We found that most HS1 knockdown B cells did not reposition their centrosome at the cell-bead interface (Fig. 5a). However, because these cells displayed not only more Arp2/3 and F-actin at the centrosome but also less Arp $2 / 3$ and F-actin at the synapse as compared with control cells, we could not exclude that impaired centrosome polarization resulted from decreased Arp2/3 and F-actin at the synapse. To address this question, we investigated the effect of Arp $2 / 3$ inhibition on centrosome polarity. We found that both Arp2/3 silencing and inhibition with CK666 had no impact on centrosome polarization to the synapse (Fig. 5b and Supplementary Fig. 5a) and, even more importantly, rescued the non-polarized phenotype of HS1-silenced activated lymphocytes (Fig. 5c and Supplementary Fig. 5b,c). Of note, Arp $2 / 3$ inhibition in these cells reduced the centrosomal pool of F-actin (Fig. 5d-f) but had no significant effect on the amounts of synapse-associated F-actin (Supplementary Fig. 5d). CK666 treatment had no impact on BCR signalling (Supplementary Fig. 5e,f). These results suggest that the centrosomal pool of Arp2/3 and F-actin prevents centrosome polarization while its synaptic counterpart is not required for this process. In support of this conclusion, a significant correlation was found between the levels of centrosomal F-actin and the distance between this organelle and the bead geometrical centre (Fig. 5g). Hence, HS1-dependent recruitment of Arp $2 / 3$ at the synapse partially depletes this complex from the centrosome, leading to a local reduction in F-actin that is needed for centrosome polarization to the synapse.

We next sought for a strategy to directly assess whether actin nucleation by Arp2/3 at the centrosome prevents the polarization of this organelle to the immune synapse. Interestingly, WASH, an actin nucleation-promoting factor that activates Arp $2 / 3$ through its VCA (verprolin homology or WH2-connector-acidic) domain, was shown to associate to the centrosome ${ }^{31}$. In agreement, in resting lymphocytes, we observed WASH as discrete punctuated structures mainly gathered around the centrosome (Supplementary Fig. 5g). In addition, in resting cells, WASH silencing decreased the amount of F-actin at the centrosome to the levels observed in activated cells (Supplementary Fig. 5h-j), indicating that it participates to local Arp2/3 activation. We therefore reasoned that targeting the WASH VCA domain to the centrosome would result in the exacerbation of local Arp2/3 activity and F-actin nucleation. Accordingly, expression of the eGFP-Centrin1-VCA fusion protein strongly increased the amount of F-actin at the centrosome (Fig. 5h,i). More importantly, expression of the eGFP-Centrin1-VCA fusion protein compromised the ability of the centrosome to polarize to the immune synapse (Fig. 5j). As observed for Arp2/3 inhibition or silencing, WASH silencing had no impact on centrosome polarity (Supplementary Fig. 5k). Consistently, centrosome polarization in eGFP-Centrin1-VCA-expressing cells was rescued by inhibiting Arp $2 / 3$ activity (Fig. 5k). These data strongly support a model where F-actin nucleation at the centrosome prevents its translocation to the synapse and must therefore be downregulated upon lymphocyte activation.

Centrosomal F-actin tethers the centrosome to the nucleus. We next searched for the cellular basis of the negative impact of Arp2/3-dependent actin nucleation at the centrosome on its ability to polarize. In view of a recent work indicating that F-actin controls centrosome positioning by inducing the retrograde transport of the nucleus in polarized fibroblasts ${ }^{32}$, we postulated that actin nucleation at the centrosome might regulate its physical interaction with the nucleus ${ }^{33}$. To test this hypothesis, we measured the distance between both organelles in lymphocytes that exhibited different levels of centrosomal 
a

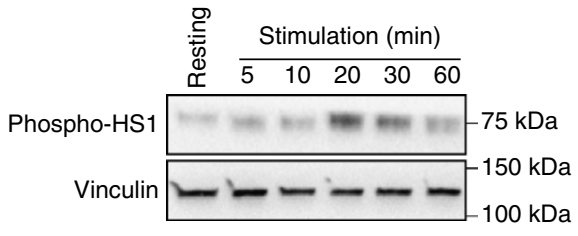

b

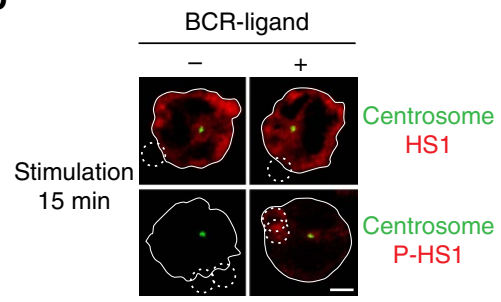

C

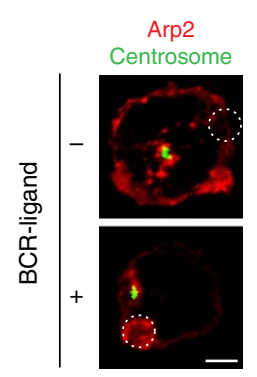

d

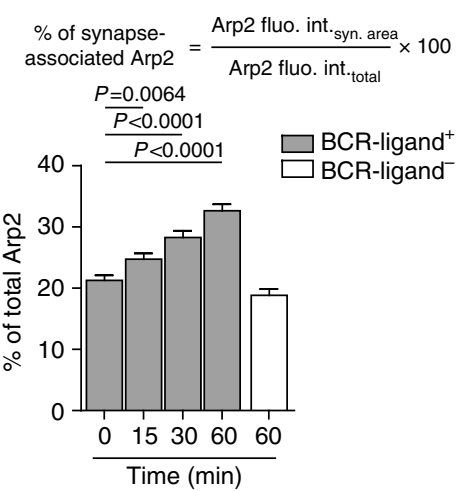

e

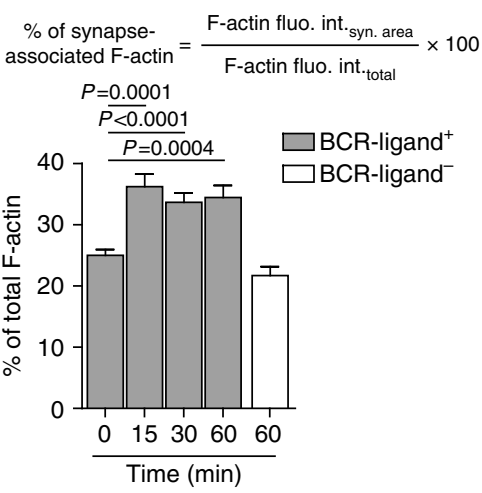

f
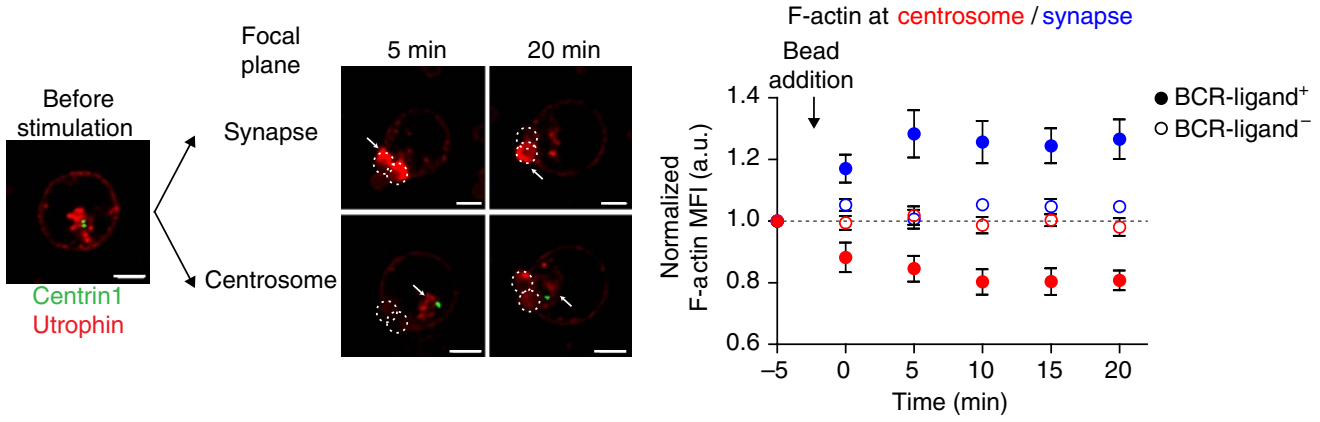

g

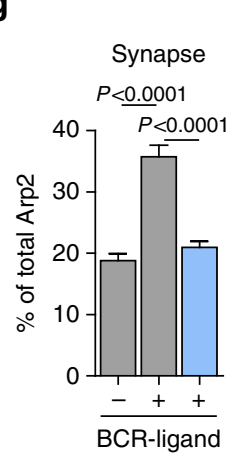

Arp2

Centrosome

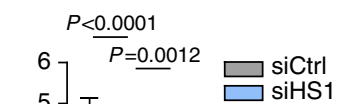

$\mathbf{h}$

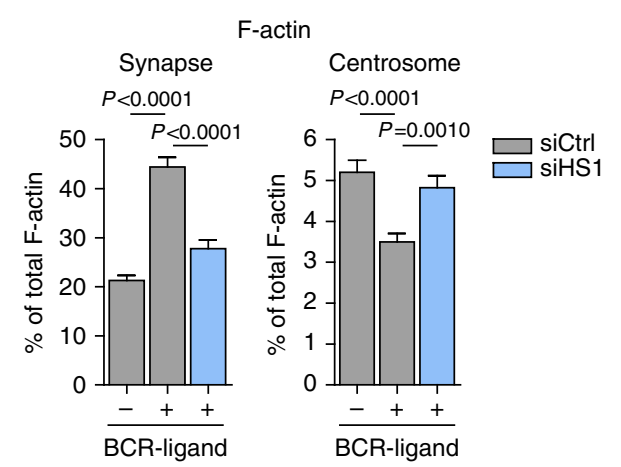

Figure 4 | Depletion of Arp2/3 from the centrosome results from its HS1-dependent recruitment at the immune synapse. (a) Western blot showing the phosphorylation of HS1 during the course of B-cell stimulation. Representative of two independent experiments. (b) Representative images of B cells stimulated with indicated beads for $15 \mathrm{~min}$, fixed and co-stained for total HS1 (top) or phosphorylated HS1 (bottom) and the centrosome ( $\alpha$-tubulin). Dashed white circles indicate bead position. Scale bar, $3 \mu \mathrm{m}$. Images are representative of two independent experiments. (c) Representative images of B cells stimulated with indicated beads for $60 \mathrm{~min}$, fixed and co-stained for Arp2 and the centrosome ( $\alpha$-tubulin). Dashed white circles indicate bead position. Scale bar, $3 \mu \mathrm{m}$. Representative of three independent experiments. (d,e) Quantification of synapse-associated Arp2 (d) and F-actin (e). (d) $n=71,64,68,72$ and 69 cells and (e) $n=55,60,66,59$ and 57 cells from left to right, pooled from three independent experiments. (f) Left: sequential images of B cells co-transfected with the centrosomal marker eGFP-Centrin1 and the F-actin probe Utrophin-RFP stimulated with BCR-ligand ${ }^{+}$beads and imaged by time-lapse spinning disk microscopy. White arrows indicate F-actin clearance from the centrosome (bottom) and its concomitant accumulation at the immune synapse (top) during the course of B-cell stimulation. Dashed white circles indicate bead position. Scale bar, $3 \mu \mathrm{m}$. Right: quantification of synapse- (blue) and centrosome- (red) associated F-actin of B cells stimulated with indicated beads. F-actin mean fluorescence intensity $(\mathrm{MFI})$ was normalized with respect to initial intensity $\left(t_{-5}\right.$ min $)$ for each cell. Data are pooled from two independent experiments and graph shows the mean \pm s.e.m. of $n=7$ cells per condition. (g,h) Quantification of Arp2 (g) and F-actin (h) associated with the synapse (left) and the centrosome (right) in control and HS1-silenced B cells stimulated for 60 min with indicated beads. Data are pooled from two (g) and three (h) independent experiments with (g) synapse: $n=51,46$ and 53 cells; centrosome: $n=51,52$ and 47 cells; and (h) synapse: $n=72,74$ and 66 cells; centrosome: $n=73,72$ and 67 cells from left to right. $P$ values determined by Mann-Whitney test. fluo. int., fluorescence intensity. syn, synapse. 
F-actin. We found that reduction of centrosomal F-actin upon BCR engagement not only stimulated centrosome polarization but was also accompanied by an increase in the distance between the nucleus and this organelle (Fig. 6a). Strikingly, such increase was equally observed when depleting or inhibiting Arp2/3 in non-stimulated cells (Fig. 6b,c), indicating that the mere reduction of centrosomal $\mathrm{F}$-actin is sufficient to induce its physical separation from the nucleus. Similarly, the distance between the centrosome and the nucleus of resting WASHsilenced cells, whose centrosome has low levels of centrosomal F-actin, was also increased (Supplementary Fig. 6a). In contrast, in activated HS1-silenced lymphocytes that maintained high levels of F-actin at their centrosome, the centrosome-nucleus distance was as short as in non-stimulated cells (Fig. 6d). This result equally applied to lymphocytes expressing the eGFPCentrin1-VCA construct, which displayed increased centrosomal F-actin and impaired centrosome polarization (Fig. 6e,f). However, centrosome-nucleus separation was rescued in these cells by reducing centrosomal F-actin with CK666 or by silencing Arp $2 / 3$ (Fig. 6 $\mathrm{d}-\mathrm{f}$ and Supplementary Fig. 6b). These results strongly suggest that the pool of F-actin at the centrosome maintains it in close proximity to the nucleus and must therefore be depleted for these two organelles to physically separate. Important molecules involved in the physical association of the centrosome to the nucleus are components of the linker of nucleoskeleton and cytoskeleton (LINC) complex. This complex includes Nesprin proteins that bind both the microtubule and actin cytoskeleton networks. We therefore investigated whether detachment of the centrosome from the nucleus as a result of LINC complex disruption might rescue centrosome polarity. Over-expression of a dominant-negative mutant of Nesprin-2 that does not bind $\mathrm{F}$-actin ${ }^{32}$ was sufficient to increase the distance between the centrosome and the nucleus in resting cells without affecting the amounts of centrosome-associated F-actin (Fig. $6 \mathrm{~g}$ and Supplementary Fig. 6c). More importantly, expression of this dominant-negative version of the LINC complex rescued both centrosome polarization and centrosome separation from the nucleus in HS1-silenced activated lymphocytes despite their high levels of centrosomal F-actin (Fig. 6h,i and Supplementary Fig. 6d,e). Hence, the need to deplete F-actin at the centrosome to detach it from the nucleus and allow its polarization to the immune synapse can be bypassed by disrupting the LINC complex. These results strongly suggest that F-actin nucleation at the centrosome is required for its physical association to the nucleus by the LINC complex. Altogether, these data further provide a putative mechanism for the need to deplete centrosomal Arp2/3 and F-actin to allow centrosome translocation to the immune synapse.

\section{Discussion}

We here found that BCR engagement with immobilized antigens induces the accumulation of the Cortactin-like protein HS1 at the immune synapse, which recruits Arp2/3, promoting the local enrichment of F-actin. Of note, although inhibition of Arp2/3 does not impair centrosome polarization, its activity is nonetheless required for $\mathrm{B}$ lymphocytes to process and present BCR-internalized antigens to $\mathrm{T}$ lymphocytes (Supplementary Fig. 6f), suggesting a function for this complex at the B-cell synapse. Recruitment of Arp2/3 at the synapse leads to its partial depletion from the centrosome, thereby reducing the pool of centrosome-nucleated F-actin. The centrosome would then be free to physically separate from the nucleus and move towards the immune synapse. Importantly, we found that an intact LINC complex is required for centrosomal F-actin to maintain centrosome attachment to the nucleus. Interestingly, although the molecular players that allow interaction between the LINC complex and the nucleoskeleton were described, the one that link this complex to the centrosome remained unclear ${ }^{33}$. Our results suggesting that F-actin nucleation at the centrosome might be a key player in this process therefore brings an interesting new piece to this puzzle.

Our findings strongly suggest that F-actin nucleation at the centrosome must decrease for this organelle to acquire a polarized localization. This might result, at least in part, from the role of centrosomal F-actin in retaining the centrosome in vicinity of the nucleus $^{33}$. The idea that the actin cytoskeleton links the centrosome to the nucleus was initially proposed in the eighties based on observations showing that nucleus purification or cell enucleation required the addition of F-actin-depolymerizing $\operatorname{drugs}^{34,35}$. However, the precise nature and origin of this actin network remain unclear. Different F-actin structures had been reported in association to the centrosome and/or the nucleus. This includes 'actin clouds' that position centrosomes and mitotic spindles $^{36}$ and resemble the F-actin structures we observed at the centrosome of $\mathrm{B}$ lymphocytes. In addition, the nucleus of migrating fibroblasts associates to a 'perinuclear actin cap'37 or to 'linear actin arrays' referred to as TAN lines, which regulate nucleus retrograde transport and centrosome polarity through the LINC complex ${ }^{32,38}$. Although we did not observe TAN lines in B lymphocytes, what might be due to their non-adherent properties, whether and how Arp2/3-dependent F-actin nucleation at the centrosome contributes to the formation and/or function of these actin structures will be an important point to investigate in the future.

Of note, we do not exclude that actin depletion at the centrosome may control additional processes required for efficient centrosome polarization than centrosome-nucleus

\footnotetext{
Figure 5 | Downregulation of centrosomal Arp2/3-dependent F-actin nucleation is required for centrosome polarization to the immune synapse. (a-c) Quantification of centrosome polarity index of control and HS1-silenced (a), CK666-treated (b) or HS1-silenced and CK666-treated (c) B cells stimulated with indicated beads for $60 \mathrm{~min}$. (a) $n=77,71$ and 75 cells; (b) $n=103,80$ and 87 cells and (c) $n=77,69,72$ and 75 cells from left to right, pooled from three independent experiments. (d) Representative images of control and HS1-silenced B cells treated with dimethylsulfoxide (DMSO) or CK666, stimulated with BCR-ligand ${ }^{+}$beads for 60 min, fixed and co-stained for F-actin (phalloidin) and the centrosome ( $\alpha$-tubulin). Scale bars, left: $3 \mu$ m; right: $0.9 \mu \mathrm{m}$. (e) Quantification of centrosome-associated F-actin from cells shown in $\mathbf{d}$ ( $n=41,47,38$ and 45 cells from left to right, pooled from two independent experiments). (f) Quantification of centrosomal F-actin of control, HS1- and HS1 plus Arp2-silenced B cells stimulated for 60 min with indicated beads ( $n=58,65,65$ and 63 cells from left to right, pooled from three independent experiments). (g) Correlation analysis of centrosomeassociated F-actin and the bead-centrosome distance $(n=185$ cells). Spearman correlation test, $P<0,0001$. (h) Left: schematics depicting the construct used to over-activate the Arp2/3 complex at the centrosome (bottom). Right: representative images of control and eGFP-Centrin1-VCA-expressing B cells, stimulated with indicated beads for $60 \mathrm{~min}$, fixed and co-stained for F-actin (phalloidin) and the centrosome (GFP). Scale bar, $3 \mu \mathrm{m}$. (d,h) White circles indicate bead position. Dashed grey squares indicate the region magnified on the right. Dashed circles on magnifications highlight the centrosomal area used for quantification. (i,j) Quantification of centrosomal F-actin (i) and centrosome polarity index (j) of cells shown in $\mathbf{h}$. (i) $n=74,66,68$ and 64 cells and $(\mathbf{j}) n=75,71$ and 64 cells from left to right, pooled from three independent experiments. (k) Quantification of centrosome polarity index of control and eGFP-Centrin1-VCA-expressing B cells, treated or not with CK666 and stimulated with indicated beads for $60 \mathrm{~min}$ ( $n=41,39,42$ and 42 cells from left to right, pooled from two independent experiments). $P$ values determined by Mann-Whitney test. Cent-VCA, eGFP-centrin1-VCA.
} 
attachment. In particular, reduction of F-actin nucleation at the centrosome might induce local changes to favour its microtubuledependent translocation to the synapse. For example, it may facilitate Dynein recruitment and/or local centrosome docking at the immune synapse. Consistent with these hypotheses, we and others have shown that Dynein is indeed required for centrosome reorientation to the synapse in both $B$ and T lymphocytes ${ }^{15,17,19,20}$. Further work is needed to unravel the role played by centrosome-nucleated F-actin in the biology of resting lymphocytes, as well as to fully understand how depletion of this F-actin pool facilitates centrosome polarity.

Our data suggest that the centrosome and the immune synapse compete for Arp2/3. Although we cannot formally exclude that there is a direct exchange between centrosomal and synaptic Arp2/3, we think that this is unlikely in view of data showing that most Arp $2 / 3$ resides in the cytosol ${ }^{39}$. We therefore propose that

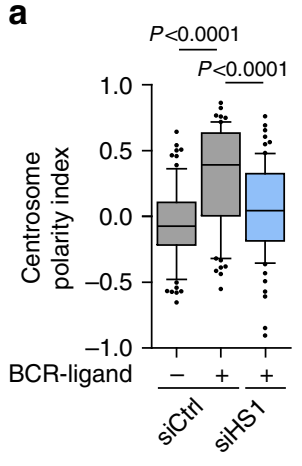

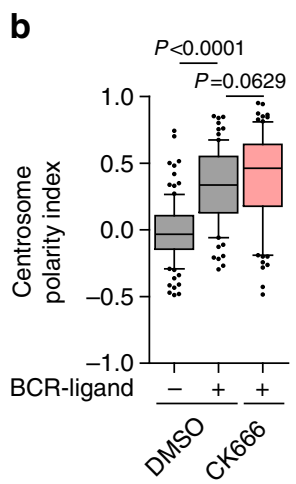

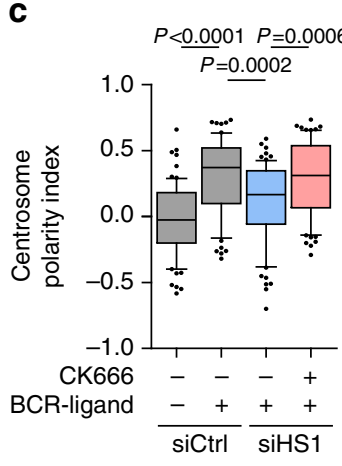

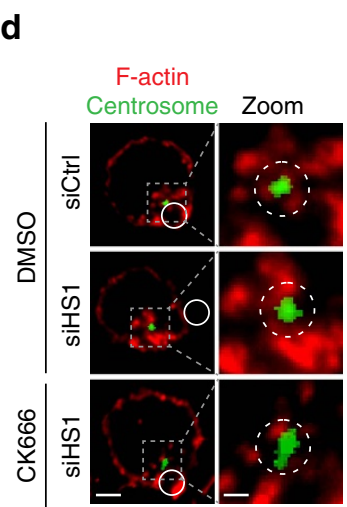

e

Centrosomeassociated F-actin

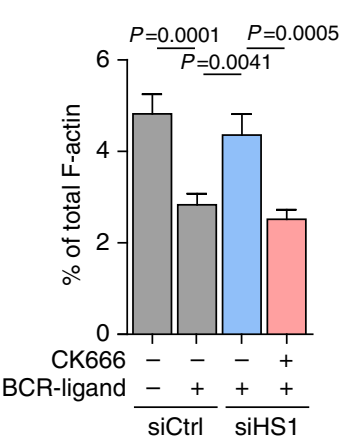

h

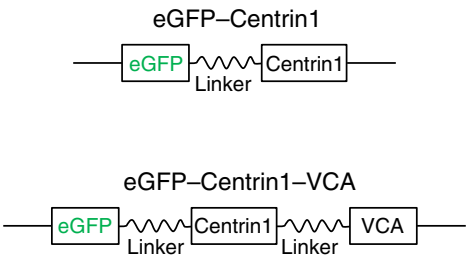

i

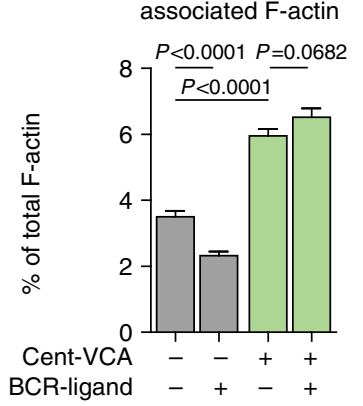

f

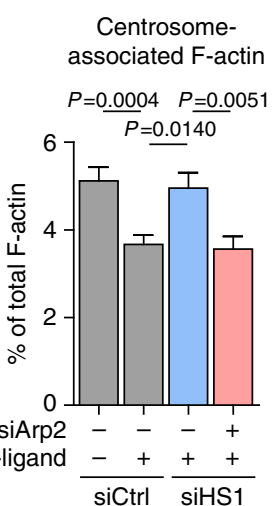

g

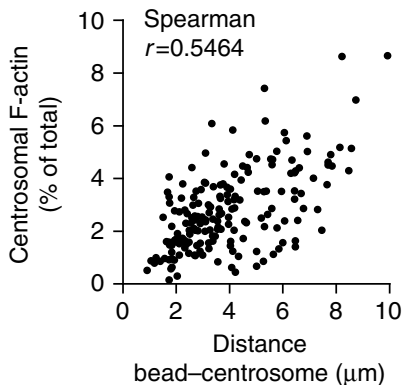

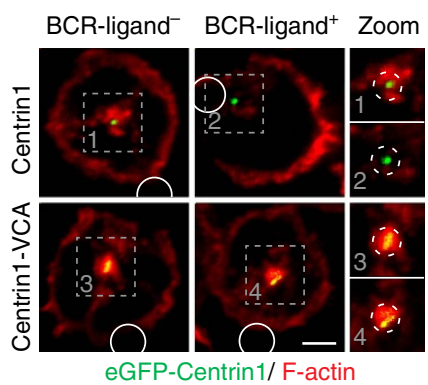

j

k
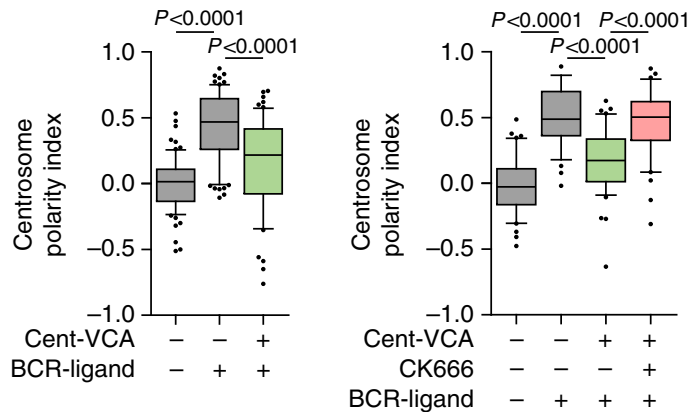
a

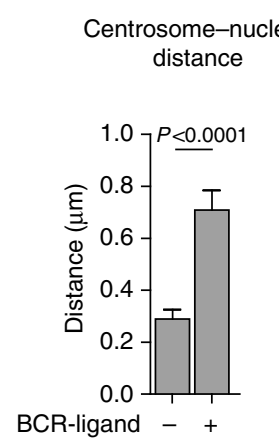

d

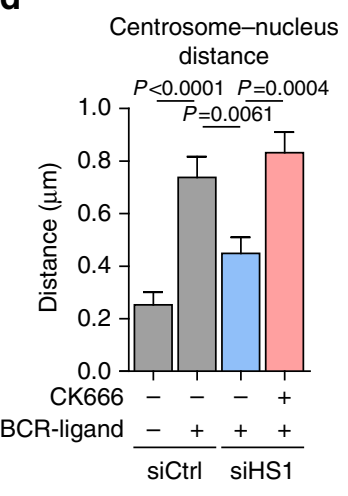

g

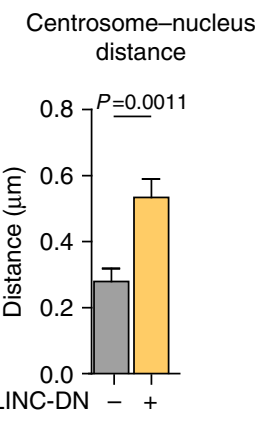

b

Centrosome-nucleus distance

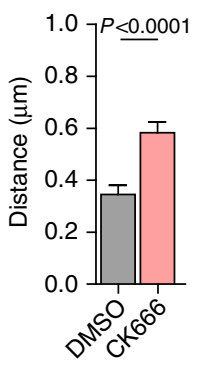

e

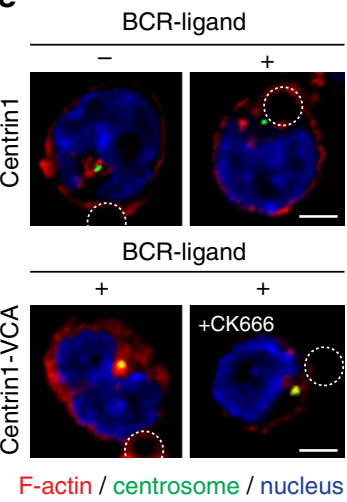

h

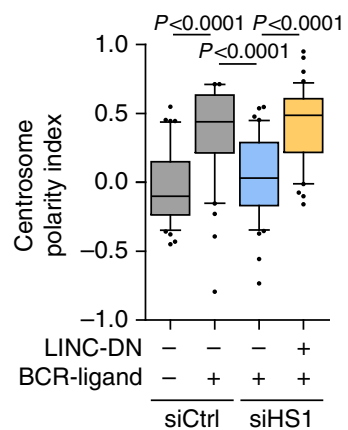

c

Centrosome-nucleus distance

$P=0.0019$

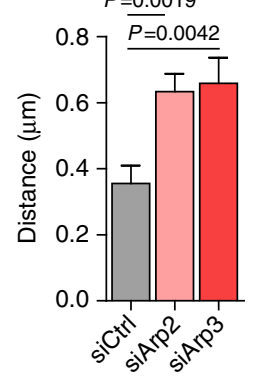

f

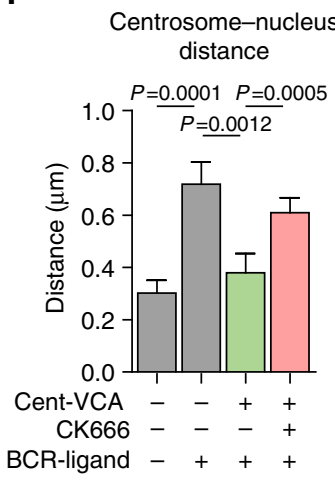

i

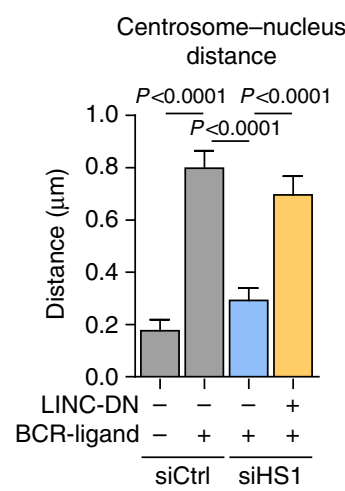

Figure 6 | Centrosomal Arp2/3-mediated F-actin nucleation links the centrosome to the nucleus through the LINC complex. (a-d) The shorter distance in three dimensions between the centrosome and the edge of the nucleus was measured in: (a) B cells stimulated with either BCR-ligand ${ }^{-}$or BCR-ligand ${ }^{+}$ beads for $60 \mathrm{~min}$; (b) resting B cells treated with dimethylsulfoxide (DMSO) or CK666 for 60 min; (c) control, Arp2- and Arp3-silenced resting B cells; and (d) control and HS1-silenced B cells, treated with either DMSO or CK666, and stimulated with indicated beads for 60 min. Data are pooled from two (c,d) and three (a,b) independent experiments with (a) $n=90$ cells per condition; (b) $n=93$ and 78 cells for DMSO and CK666, respectively; (c) $n=43,35$ and 31 cells; and (d) $n=56,53,52$ and 54 cells from left to right. (e) Representative images of B cells over-expressing the eGFP-Centrin1 protein or the eGFP-Centrin1-VCA fusion protein treated or not with CK666, stimulated with indicated beads for 60 min, fixed and co-stained for F-actin (phalloidin), the centrosome (GFP) and the nucleus (DAPI). Dashed circles indicate bead position. Scale bar, $3 \mu \mathrm{m}$. (f) Quantification of the distance between the centrosome and the nucleus edge from cells shown in e. Data are pooled from two independent experiments with $n=41,39,42$ and 43 cells from left to right. (g) Quantification of the distance between the nucleus edge and the centrosome in resting B cells over-expressing or not the LINC-DN construct. Data are pooled from three independent experiments with $n=64$ cells per condition. (h,i) Quantification of centrosome polarity index (h) and centrosomenucleus distance (i) of control and HS1-silenced B cells, over-expressing or not the LINC-DN construct and stimulated for 60 min with indicated beads. Data are pooled from two independent experiments with $(\mathbf{h}) n=41,40,42$ and 42 cells and (i) $n=41,44,41$ and 45 cells from left to right. $P$ values determined by Mann-Whitney test. DAPI, 4',6-diamidino-2-phenyindole.

both the centrosome and the synapse are rather competing for the cytosolic pool of Arp2/3.

Anyhow, our data strongly suggest that there is an effective competition between distinct subcellular locations for this actin-nucleating complex. As a result of this competition, cells would respond to extracellular stimuli by favouring one biological process over another, what might provide a simple mechanism for cells to coordinate them in time and space. In addition, the existence of a competition for cytosolic Arp $2 / 3$ implies that the pool of centrosome-associated Arp2/3 might be minor in adherent cells as compared with lymphocytes, given that they use this complex to form adhesive structures such as lamellipodia. 
Interestingly, it has been shown that immune synapses share many features with lamellipodia ${ }^{40}$, suggesting that the competition between the centrosome and the immune synapse for Arp2/3 might also apply to the centrosome and the lamellipodium of migrating cells.

In conclusion, our results highlight an unexpected role for the regulation of centrosome-associated F-actin in the control of lymphocyte polarization. Whether and how this novel regulatory mechanism applies to additional biological systems that rely on cell polarity is an open question. This would be of particular interest in the context of cilium biogenesis that involves signalling pathways also used in immune synapse formation by lymphocytes ${ }^{41,42}$.

\section{Methods}

Cells and cell culture. The mouse $\mathrm{IgG}^{+}$B-lymphoma cell line IIA1.6 (derived from the A20 cell line (American Type Culture Collection \#: TIB-208)) and the LMR7.5 T-cell hybridoma that recognizes I-A ${ }^{\mathrm{d}}$-Lack ${ }_{156-173}$ complexes (from and described in ref. 43) were cultured as reported ${ }^{14}$ in CLICK medium (RPMI 1640-GlutaMax-I supplemented with $10 \%$ fetal calf serum, $1 \%$ penicillinstreptomycin, $0.1 \% \beta$-mercaptoethanol and $2 \%$ sodium pyruvate). All cell culture products were purchased from GIBCO/Life Technologies. All experiments were conducted in 50\% CLICK/50\% RPMI 1640-GlutaMax-I.

SiRNA. Protein silencing was achieved using the Neon transfection system (Invitrogen, Life Technologies). Briefly, B cells were washed in phosphate-buffered saline (PBS), resuspended in Buffer R at a density of $50 \times 10^{6}$ cells per $\mathrm{ml}$ and ON-TARGETplus SMARTpool Non-targeting (siCtrl), HS1- (siRNA\#1: 5'-GGG CAUGAUGUAUCGGUUU-3'; siRNA\#2: 5' -CCAAGGAGAGGGAAGCGAU-3'; siRNA\#3: $5^{\prime}$-UGGAAGAGCCAGUGUACGA-3'; and siRNA\#4: $5^{\prime}$-GUAAAGAU GAGCCGAGAAG- $3^{\prime}$ ), Arp2- (siRNA\# 1: 5' -UGGUGUAACUGUUCGAUAA- $3^{\prime}$; siRNA\#2: $5^{\prime}$-GUUCUUUACUAAUGACGAA-3'; siRNA\#3: $5^{\prime}$-GAUCAGUGC UUCUCGACAA-3'; and siRNA\#4: 5'-CAUCGAGGUUGGAACGAGA), Arp3(siRNA\#1: $5^{\prime}$-GAAGAGAGCUAAGACGAUU-3'; siRNA\#2: $5^{\prime}$-AAGCAGUGAA GGAACGCUA-3'; siRNA\#3: 5'-GCUGACGGGUACAGUAAUA; siRNA\#4: $5^{\prime}-\mathrm{G}$ AGUCAACGCCAUCUCAAA) or WASH- (siRNA\# 1 : $5^{\prime}$-ACAGCAACACGGC GGAAUA- ${ }^{\prime}$; siRNA\#2: $5^{\prime}$-GAGGAGAAAUUGUUCGAUG- ${ }^{\prime}$; siRNA\#3: $5^{\prime}$-GC ACAUUCAGGAACGUUUA- ${ }^{\prime}$; and siRNA\#4: $5^{\prime}$-GAAUACGGCUCCAUCUU UA- ${ }^{\prime}$ ) targeting siRNA (Dharmacon, GE Healthcare) were added at a final concentration of $200 \mathrm{nM}$. Cells were then electroporated $(1,300 \mathrm{~V}, 2$ pulses, $20 \mathrm{~ms}$ per pulse) using 10- $\mu$ l tips, and incubated in CLICK medium for 60-72 h. Silencing efficiency was analysed by western blot as described below.

Plasmids. The eGFP-Centrin 1 and Utrophin-RFP plasmids were obtained from M. Bornens and M. Piel, respectively (Institut Curie, Paris, France). The eGFP-Centrin1-VCA plasmid was obtained by sub-cloning in frame the VCA domain of WASH at the C terminus of the eGFP-Centrin1 construct. Briefly, the Centrin 1 cDNA deleted from its stop codon was amplified from the eGFP-Centrin 1 plasmid using the following primers: forward:

$5^{\prime}$-ctaggtaccatggcttccggcttcaaga- $3^{\prime}$ and reverse: $5^{\prime}$-gcaggatccgtaaaggctggtcttcttcat- $3^{\prime}$ (which include KpnI and BamHI restriction sites (underlined), respectively). The WASH VCA cDNA was amplified from a previously home-made (A. Gautreau) WASH plasmid by using the primers: forward: $5^{\prime}$-ttaggatccTCCGGACTCAGAT CTCGAGCTCAAGCTTCGAATTCTGCAGTCGACcagggagcccctaagga-3' (which includes BamHI restriction site (underlined) and the sequence for a peptide linker (upper case)) and reverse: $5^{\prime}$-gattctagaTCAggactcccagtcctcct- $3^{\prime}$ (which includes XbaI restriction site (underlined) and a stop codon (upper case)). Both fragments were then sub-cloned in frame within the original vector using KpnI and XbaI restriction enzymes. BamHI restriction enzyme was used to orient the two fragments. The resulting eGFP-Centrin1-VCA plasmid was sequenced to ensure sequence integrity. The LINC-DN (Nesprin-2 SRKASH) plasmid was obtained from E.R. Gomes and described in ref. 32. Plasmid expression was achieved by electroporating $2 \times 10^{6}$ B-lymphoma cells, with 1 or $3 \mu \mathrm{g}$ of plasmid using the Amaxa Cell Line Nucleofactor Kit R (T-016 programme, Lonza). Cells were incubated in CLICK medium for $16-20 \mathrm{~h}$ before analysis.

Reagents. The Lack antigen was produced and purified by the 'Recombinant Protein' platform (UMR144, Institut Curie, Paris, France), and Lack peptide (aa 156-173) was synthetized by PolyPeptide Group. Cytochalasin D and nocodazole used from centrosome purification were from Sigma Aldrich. For inhibition of Arp2/3 activity, cells were pretreated with $25 \mu \mathrm{M}$ CK666 (Tocris Bioscience) for $30 \mathrm{~min}$ before being stimulated for indicated time in presence of the drug.

Antibodies. The following primary antibodies were used for immunofluorescence: rabbit anti- $\gamma$-tubulin (Abcam, \#Ab11317, 1/2000); fluorescein isothiocyanate
(FITC)-conjugated mouse anti- $\alpha$-tubulin (Abcam, \#Ab64503, 1/100); rabbit anti-Arp2 (Abcam, \#Ab47654, 1/200); rabbit anti-HS1 and rabbit anti-phosphoHS1 (both from Cell Signalling Technologies, \#4557S and \#8714P, respectively, $1 / 50$ ); rabbit anti-WASH (home-made as previously described ${ }^{44}, 1 / 250$ ); and human anti-green fluorescent protein (GFP) and anti-red fluorescent protein (RFP) (Recombinant Antibodies Platform, Institut Curie, Paris, France, 1/200). The following secondary antibodies were used: AlexaFluor488-, AlexaFluor568-, Cy3-, Cy5- and AlexaFluor647-conjugated $\mathrm{F}\left(\mathrm{ab}^{\prime}\right)_{2}$ donkey anti-rabbit (Jackson ImmunoResearch, 1/300); and AlexaFluor488- and Cy3- conjugated donkey antihuman (Life Technologies and Jackson ImmunoResearch, respectively, 1/200). F-actin was stained using AlexaFluor546- or AlexaFluor647-conjugated phalloidin (Life Technologies, \#A22283 and \#A22287, respectively, 1/100). Nuclei were stained using 4',6-diamidino-2-phenyindole (DAPI, Sigma Aldrich, 1/5,000).

For western blotting, the following antibodies were used: mouse anti- $\gamma$-tubulin (Sigma Aldrich, \#T6557-.2ML, 1/500); rabbit anti-HS1, phospho-HS1 and phospho-Erk (Cell Signaling Technologies, \#4557S, \#8714P and \#4377S, respectively, 1/1,000); rabbit anti-Arp2 (Abcam, \#Ab47654, 1/200); rabbit anti-WASH (home-made as previously described ${ }^{44}, 1 / 250$ ); and mouse anti-Arp3 and anti-vinculin (Sigma Aldrich, \#A5979-200UL, 1/200, and \#V9264-200UL, $1 / 1,000$, respectively), followed by horseradish peroxidase-conjugated donkey anti-mouse or rabbit (Jackson ImmunoResearch, 1/5,000).

Preparation of BCR-ligand-coated beads. In all, $4 \times 10^{7} 3$ - $\mu \mathrm{m}$ latex $\mathrm{NH}_{2}$-beads (Polyscience) were activated with $8 \%$ glutaraldehyde (Sigma Aldrich) for $2 \mathrm{~h}$ at room temperature. Beads were washed with PBS $1 \mathrm{x}$ and incubated overnight at $4{ }^{\circ} \mathrm{C}$ with different ligands: $100 \mu \mathrm{g} \mathrm{ml}^{-1}$ of either $\mathrm{F}\left(\mathrm{ab}^{\prime}\right)_{2}$ goat anti-mouse IgG (BCR-ligand ${ }^{+}$ beads) or $\mathrm{F}\left(\mathrm{ab}^{\prime}\right)_{2}$ goat anti-mouse IgM (BCR-ligand ${ }^{-}$beads; MP Biomedical) in combination or not with $100 \mu \mathrm{g} \mathrm{ml}^{-1}$ of the Leishmania major antigen Lack.

B-cell stimulation and immunofluorescences. Cells were plated on poly-L-lysinecoated slides and stimulated with indicated beads at a 1:2 ratio (cell:beads) for different time at $37^{\circ} \mathrm{C}$ and fixed in $4 \%$ paraformaldehyde for $12 \mathrm{~min}$ at room temperature. For $\gamma$-tubulin staining, cells were further incubated with ice-cold $100 \%$ methanol for $2 \mathrm{~min}$ and quenched for $10 \mathrm{~min}$ with PBS/100 mM glycine. Fixed cells were incubated $45-60$ min with primary antibodies and $30 \mathrm{~min}$ with secondary antibodies in PBS-BSA-Saponin $(1 \mathrm{x} / 0.2 \% / 0.05 \%)$

Time-lapse imaging. A total of $1 \times 10^{5}$ B-lymphoma cells co-expressing the centrosomal marker eGFP-Centrin 1 and the F-actin probe Utrophin-RFP were seeded in 35-mm FD35 Fluorodish (World Precision Instruments, Inc). Cells were either treated with dimethylsulfoxide (DMSO) or CK666 $(25 \mu \mathrm{M})$ or stimulated with either BCR-ligand ${ }^{-}$or BCR-ligand ${ }^{+}$beads and recorded at $37^{\circ} \mathrm{C}, 5 \% \mathrm{CO}_{2}$ using an inverted spinning disk confocal microscope (Roper/Nikon) equipped with a $\times 60$ (1.4 numerical aperture (NA)) oil immersion objective and a CoolSNAP HQ2 camera. The images were acquired every 5 min with $z$-stack of $1 \mu \mathrm{m}$. For the analysis, Fiji (ImageJ) software was used to reconstruct the three-dimensional (3D) movies, correct bleaching (exponential fit correction) and analyse the amount of F-actin associated with the centrosome and the synapse focal plane.

Stable isotope labelling by amino acids in cell culture. IIA1.6 cells were maintained in L-lysine-depleted SILAC RPMI 1640 (Thermo Scientific, Life Technologies) supplemented with $10 \%$ dialysed $\mathrm{FBS}$ and $0.1 \mathrm{mg} \mathrm{ml}^{-1}$ heavy $\left[{ }^{13} \mathrm{C}_{6}\right.$ or light $\left[{ }^{12} \mathrm{C}_{6}\right]$ L-lysine (Thermo Scientific, Life Technologies). Every 3-4 days, cells were split and media replaced with the corresponding light- or heavy-labelling medium. After six to seven cell divisions, cells achieved $\geq 96 \%$ incorporation of amino-acid isotopes.

Centrosome purification. Centrosomes were purified as previously described ${ }^{45}$ with slight modifications. Briefly, following stimulation with indicated beads for $60 \mathrm{~min}$, cells were incubated on ice with $200 \mathrm{nM}$ nocodazole and $1 \mu \mathrm{g} \mathrm{ml}^{-1}$ cytochalasin D for $90 \mathrm{~min}$. Cells were washed and lysed in TicTac buffer (16 mM PIPES, $10 \mathrm{mM}$ HEPES (pH 7.5), $50 \mathrm{mM} \mathrm{KCl}, 1.2 \mathrm{mM}$ EGTA, $5 \mathrm{mM} \mathrm{MgCl}_{2}, 0.1 \%$ Triton X-100 and $0.1 \% \beta$-mercaptoethanol) for $15 \mathrm{~min}$. Centrosomes were isolated by sequential centrifugations at (1) $10,000 \mathrm{~g}$ for $30 \mathrm{~min}$ at $4{ }^{\circ} \mathrm{C}$ on top of a $60 \% \mathrm{w} / \mathrm{v}$ sucrose cushion and (2) $40,000 \mathrm{~g}$ for $60 \mathrm{~min}$ at $4{ }^{\circ} \mathrm{C}$ on top of a discontinuous sucrose gradient $(40-50-70 \%$, w/w). Finally, 10 fractions of $0.5 \mathrm{ml}$ were recovered from the bottom of the tube, and centrosome-containing fractions were identified by western blot.

Proteomics. Sample preparation. Proteins from centrosome preparations were separated on $10 \%$ SDS-PAGE gels (Invitrogen) and stained with colloidal blue staining (LabSafe GEL BlueTM GBiosciences). Gel slices were excised (20 fractions) and proteins were reduced with $10 \mathrm{mM}$ dithiothreitol before alkylation with $55 \mathrm{mM}$ iodoacetamide. After washing and shrinking of the gel fractions with $100 \% \mathrm{MeCN}$, in-gel digestion was performed using recombinant endoproteinase rLys-C (Promega) overnight in $25 \mathrm{mM} \mathrm{NH}_{4} \mathrm{HCO}_{3}$ at $30^{\circ} \mathrm{C}$.

MS analysis. Peptides were extracted and analysed by nano-LC-MS/MS using an Ultimate 3000 system (Dionex S.A.) coupled to a LTQ-Orbitrap XL mass spectrometer (Thermo Fisher Scientific), as described ${ }^{46}$. Samples were loaded on a 
C18 pre-column ( $300 \mu \mathrm{m}$ inner diameter $\times 5 \mathrm{~mm}$; Dionex) at $20 \mu \mathrm{min}^{-1}$ in $5 \%$ MeCN and $0.1 \%$ TFA. After 3 min of desalting, the pre-column was switched on the C18 column $(75 \mu \mathrm{m}$ inner diameter $\times 15$ or $50 \mathrm{~cm}$, packed with C18 PepMap, $3 \mu \mathrm{m}, 100 \AA ̊$; LC Packings) equilibrated in solvent A ( $5 \% \mathrm{CH}_{3} \mathrm{CN}$ and $0.1 \%$ $\mathrm{HCOOH}$ ). Bound peptides were eluted using a $97-\mathrm{min}$ linear gradient (from 5 to $30 \%(\mathrm{v} / \mathrm{v}))$ of solvent $\mathrm{B}\left(80 \% \mathrm{CH}_{3} \mathrm{CN}\right.$ and $\left.0.085 \% \mathrm{HCOOH}\right)$ at a $150-\mathrm{nl} \mathrm{min}{ }^{-1}$ flow rate and oven temperature of $40^{\circ} \mathrm{C}$. Data-dependent acquisition was performed on the LTQ-Orbitrap mass spectrometer in the positive-ion mode. Survey MS scans were acquired in the Orbitrap on the $480-1,200 \mathrm{~m} / \mathrm{z}$ range with the resolution set to a value of 60,000 . Each scan was recalibrated in real time by co-injecting an internal standard from ambient air into the C-trap (lock mass option). The five most intense ions per survey scan were selected for collisioninduced dissociation (CID) fragmentation and the resulting fragments were analysed in the linear trap (LTQ). Target ions already selected for MS/MS were dynamically excluded for $180 \mathrm{~s}$.

Data analysis. Data were acquired using the Xcalibur software (v2.0.7) and the resulting spectra were analysed via the Mascot Software (v2.3) with Proteome Discoverer (v1.2, Thermo Scientific) using the SwissProt Mus musculus database. Carbamidomethylation of cysteine, oxidation of methionine, $\mathrm{N}$-terminal acetylation and heavy ${ }^{13} \mathrm{C}_{6}$-lysine (Lys6) were set as variable modifications. We set specificity of trypsin digestion and allowed two missed cleavage sites and mass tolerances in MS, and MS/MS were set to 2 p.p.m. and $0.8 \mathrm{Da}$, respectively. The resulting Mascot result files were further processed using $m y \operatorname{ProMS}^{47}$ (v3.0), allowing a maximum FDR of $1 \%$ by automatically filtering the Mascot score at the peptide level.

Protein quantification. For SILAC-based protein quantification, peptides XICs (extracted ion chromatograms) were retrieved from Proteome Discoverer. Scale normalization computed using the 'package limma' from $\mathrm{R}$ was applied to compensate for mixing errors of the different SILAC cultures as described ${ }^{48}$ Protein ratios were computed as the geometrical mean of related peptides. To estimate ratio significance, a $t$-test was performed with a Benjamini-Hochberg FDR control threshold set to 0.05 . All quantified proteins have at least three peptides quantified (all peptides selected). Peptide intensity ratio outliers were removed when their value was too far from the median observed in the peptide intensity ratio set for a given protein. Protein quantification ratio outliers were not computed when the identified peptide number was too different between the two channels. Proteins displaying a minimal absolute fold change $\geq 10 \%$ that reaches statistical significance (adjusted $P$ value of quantification $\leq 0.05$ ) were considered as differentially associated with the centrosome of activated lymphocytes. This led to the selection of 835 proteins.

GO term enrichment analysis. Protein analysis by GO term enrichment was computed based on annotation only and did not take into account the relative abundance of the 835 proteins in resting and activated lymphocytes. The frequency of each GO was computed in the Mus musculus proteome (defined as the background, Slim Ontology file including all 21,283 mouse proteins) and compared with the set. We reported only the GO terms with the frequency statistically enriched in our protein set compared with the background. GO enrichment factors were computed with the GO::TermFinder ${ }^{49}$ through $m y$ ProMS. Briefly, to determine whether any GO term annotates a specified list of proteins at a frequency greater than the one expected by chance, GO::TermFinder calculates a $P$ value using a hyper-geometric distribution. For multiple testing corrections, FDR was controlled and set to $1 \%$ (Benjamini-Hochberg). A $P$ value was associated to each GO term individually. The FDR corresponds to the cutoff applied to the list of all the GO terms.

In vitro nucleation assays. In vitro nucleation assays were performed according to Farina et al. ${ }^{26}$. In brief, centrosomes isolated in TicTac buffer were incubated for $20 \mathrm{~min}$ on surface of a polydimethylsiloxane open chamber. The excess of centrosomes was washed with TicTac buffer supplemented with $1 \%$ BSA (TicTac-BSA). Microtubule and actin nucleation was induced by diluting tubulin dimers (labelled with ATTO-565, $30 \mu \mathrm{M} \mathrm{final}^{26}$ ) or actin monomers (labelled with Alexa-568, $1 \mu \mathrm{M}$ final ${ }^{50-52}$ ) in TicTac buffer supplemented with $1 \mathrm{mM}$ GTP, $2.7 \mathrm{mM}$ ATP, $10 \mathrm{mM}$ dithiothreitol, $20 \mu \mathrm{g} \mathrm{ml}^{-1}$ catalase, $3 \mathrm{mg} \mathrm{ml}^{-1}$ glucose, $100 \mu \mathrm{g} \mathrm{ml}^{-1}$ glucose oxidase and $0.25 \% \mathrm{w} / \mathrm{v}$ methylcellulose. In addition, a threefold molar equivalent of profilin to actin was added in the reaction mixture ${ }^{53}$. Time-lapse observations were performed by using a total internal reflection fluorescence microscope (Roper Scientific) equipped by an iLasPulsed system and an Evolve camera (EMCCD $512 \times 512$, pixel $=16 \mu \mathrm{m}$ ) using a $\times 601.49 \mathrm{NA}$ objective lens. For quantification of the actin nucleation activity by centrosomes, F-actin fluorescence intensity was integrated over a 2 - $\mu \mathrm{m}$-diameter circle around the centrosome and normalized with respect to initial intensity. $\gamma$-Tubulin staining, needed for the efficiency calculation, was performed at the end of the movie recording under the microscope without prior fixation. Primary and secondary antibodies, diluted in TicTac-BSA buffer, were incubated for 60 and $30 \mathrm{~min}$, respectively. Arp $2 / 3$ complex inhibition experiments were performed by adding $200 \mu \mathrm{M}$ CK666 in reaction mixture. dimethylsulfoxide was used as control. Immunofluorescence staining of isolated centrosomes was performed by incubating centrosomes, seeded on a clean surface, with primary and secondary antibodies for 60 and $30 \mathrm{~min}$, respectively.

Western blotting. B cells were lysed at $4^{\circ} \mathrm{C}$ in RIPA buffer (Thermo Scientific) supplemented with $1 \times$ protease inhibitor cocktail (Roche) and $1 \times$ Halt phosphatase inhibitor cocktail (Thermo Scientific). Supernatants were collected and loaded onto mini-PROTEAN TGX SDS-PAGE gels and transferred onto polyvinylidene fluoride membrane (Trans-Blot Turbo Transfer). Membranes were blocked in 5\% non-fat dry milk resuspended in $1 \times$ TBS- $0.05 \%$ Tween- 20 and incubated overnight at $4{ }^{\circ} \mathrm{C}$, with primary antibodies followed by $60 \mathrm{~min}$ incubation with secondary antibodies. Western blots were developed with Clarity Western ECL substrate, and chemiluminescence was detected using the ChemiDoc imager (all from BioRad). Full scans of unprocessed western blots are available in Supplementary Fig. 7.

Calcium measurement. A total of $1 \times 10^{6}$ B cells were loaded with $1 \mu \mathrm{M}$ Fluo- 4 AM and Fura Red AM (Life Technologies) for $15 \mathrm{~min}$ at $37^{\circ} \mathrm{C}$ in RPMI 1640 and resuspended in CLICK medium. The fluorescence of Fluo-4 and Fura Red were analysed using a BD Accuri C6 flow cytometer (BD Biosciences). After 120-s recording to assess basal $\mathrm{Ca}^{2+}$ levels, BCR-ligand at a final concentration of $10 \mathrm{\mu g} \mathrm{ml}^{-1}$ was added to the cell suspension and the $\mathrm{Ca}^{2+}$ levels were measured for $300 \mathrm{~s}$. Finally, the ratio Fluo-4/Fura Red and the geometric mean over time were calculated using FlowJo software (v10, BD Biosciences).

Antigen presentation. B cells were incubated with Lack \pm BCR-ligand-coated beads for $5 \mathrm{~h}$ or with peptide control for $1 \mathrm{~h}$. Cells were washed with PBS, fixed in ice-cold PBS $/ 0.01 \%$ glutaraldehyde for $1 \mathrm{~min}$ and quenched with $\mathrm{PBS} / 100 \mathrm{mM}$ glycine. B cells were then incubated with Lack-specific T-cell hybridoma in a 1:1 ratio for $24 \mathrm{~h}$. Supernatants were collected and interleukin-2 cytokine production was assessed using BD optEIA Mouse IL-2 ELISA set following the manufacturer's instructions (BD Biosciences).

Immunofluorescence acquisition and analysis. All $z$-stack images $(0.5-\mu \mathrm{m}$ spacing) were acquired on an inverted spinning disk confocal microscope (Roper/ Nikon) with a $\times 60 / 1.4 \mathrm{NA}$ oil immersion objective. Image processing was performed with Fiji (ImageJ) software ${ }^{54}$. Because methanol fixation required for $\gamma$-tubulin staining is not compatible with phalloidin labelling, the centrosome was stained with antibodies directed against $\alpha$-tubulin, and a threshold was applied to visualize the centrosome but not the microtubules as described in Supplementary Fig. 2d. Single-cell images shown in the figures were cropped from large fields, rotated and their contrast and brightness manually adjusted. Images shown are the average $z$-projection of three planes around the centrosome.

Centrosome polarity index was computed as described in Fig. 1a. Briefly, $z$-stacks were projected (SUM slice) and images were automatically threshold (Default) on the green channel to obtain the centre of mass of the cell (Cell $\left.{ }_{\mathrm{CM}}\right)$. Then, the position of the centrosome and the bead geometrical centre $\left(\right.$ Bead $\left._{\mathrm{GC}}\right)$ were manually selected. The position of the centrosome was then projected $\left(\mathrm{Cent}_{\mathrm{proj}}\right)$ on the vector defined by the $\mathrm{Cell}_{\mathrm{CM}}-\mathrm{Bead}_{\mathrm{GC}}$ axis. The centrosome polarity index was calculated by dividing the distance between the $\mathrm{Cell}_{\mathrm{CM}}$ and the Cent $t_{\text {proj }}$ by the distance between the Cell $\mathrm{CM}_{\mathrm{CM}}$ and the $\mathrm{Bead}_{\mathrm{GC}}$. The index ranges from -1 (anti-polarized) to 1 (fully polarized).

Centrosome- and synapse-associated Arp2 and F-actin were quantified as shown in Fig. 2b and Supplementary Fig. 4a, respectively. Briefly, after manual selection of the centrosome, background subtraction (rolling ball $50 \mathrm{px}$ ) on the $z$-projection (AVG) of the three planes around the centrosome was performed. We then computed the radial distribution of cytoplasmic Arp2 and F-actin fluorescence intensities from the centrosome of resting cells. The drop in fluorescence intensities (at $0.8 \mu \mathrm{m}$ from the centrosome) was used as a threshold to define the radius of the centrosomal area that was further used to assess the amount of Arp2 and F-actin associated with the centrosome. The synaptic area was manually defined by positioning a fixed area at the cell-bead interface.

The distance between the centrosome and the nucleus was measured in three dimensions. For this, the nucleus was automatically threshold in 3D (Otsu) and the corresponding 3D-distance map was computed (Image 3D suite plugin). The 3D position of the centrosome was then manually selected on the cell stack and the shorter distance to the nucleus edge was measured on the 3D-distance map.

Statistics. All graphs and statistical analysis were performed with GraphPad Prism 5 (GraphPad Software). No statistical method was used to predetermine sample size. Kolmogorov-Smirnov test was used to assess normality of all data sets. Mann-Whitney test was used to determine statistical significance excepted when mentioned. Boxes in box plots extend from the 25th to 75th percentile, with a line at the median and whiskers extend from the 10th to the 90th percentile. Bar graphs show the mean \pm s.e.m.

\section{References}

1. Barnett, B. E. et al. Asymmetric B cell division in the germinal center reaction. Science 335, 342-344 (2012).

2. Thaunat, O. et al. Asymmetric segregation of polarized antigen on B cell division shapes presentation capacity. Science 335, 475-479 (2012).

3. Schmoranzer, J. et al. Par3 and dynein associate to regulate local microtubule dynamics and centrosome orientation during migration. Curr. Biol. 19, 1065-1074 (2009). 
4. Elric, J. \& Etienne-Manneville, S. Centrosome positioning in polarized cells: common themes and variations. Exp. Cell Res. 328, 240-248 (2014).

5. Yuseff, M. I., Pierobon, P., Reversat, A. \& Lennon-Dumenil, A. M. How B cells capture, process and present antigens: a crucial role for cell polarity. Nat. Rev. Immunol. 13, 475-486 (2013).

6. del Pozo, M. A. et al. The two poles of the lymphocyte: specialized cell compartments for migration and recruitment. Cell Adhes. Commun. 6, 125-133 (1998).

7. Chang, J. T. et al. Asymmetric T lymphocyte division in the initiation of adaptive immune responses. Science 315, 1687-1691 (2007).

8. Stinchcombe, J. C. \& Griffiths, G. M. Communication, the centrosome and the immunological synapse. Philos. Trans. R. Soc. Lond. B. Biol. Sci. 369, 20130463 (2014).

9. Kupfer, A., Swain, S. L. \& Singer, S. J. The specific direct interaction of helper $\mathrm{T}$ cells and antigen-presenting B cells. II. Reorientation of the microtubule organizing center and reorganization of the membrane-associated cytoskeleton inside the bound helper T cells. J. Exp. Med. 165, 1565-1580 (1987).

10. Harwood, N. E. \& Batista, F. D. The cytoskeleton coordinates the early events of B-cell activation. Cold Spring Harb. Perspect. Biol. 3, a002360 (2011).

11. Rak, G. D., Mace, E. M., Banerjee, P. P., Svitkina, T. \& Orange, J. S. Natural killer cell lytic granule secretion occurs through a pervasive actin network at the immune synapse. PLoS Biol. 9, e1001151 (2011).

12. Ritter, A. T. et al. Actin depletion initiates events leading to granule secretion at the immunological synapse. Immunity 42, 864-876 (2015).

13. Stinchcombe, J. C., Majorovits, E., Bossi, G., Fuller, S. \& Griffiths, G. M. Centrosome polarization delivers secretory granules to the immunological synapse. Nature 443, 462-465 (2006).

14. Yuseff, M. I. et al. Polarized secretion of lysosomes at the B cell synapse couples antigen extraction to processing and presentation. Immunity 35, 361-374 (2011).

15. Liu, X., Kapoor, T. M., Chen, J. K. \& Huse, M. Diacylglycerol promotes centrosome polarization in T cells via reciprocal localization of dynein and myosin II. Proc. Natl Acad. Sci. USA 110, 11976-11981 (2013).

16. Quann, E. J., Liu, X., Altan-Bonnet, G. \& Huse, M. A cascade of protein kinase $\mathrm{C}$ isozymes promotes cytoskeletal polarization in T cells. Nat. Immunol. 12, 647-654 (2011).

17. Reversat, A. et al. Polarity protein Par3 controls B-cell receptor dynamics and antigen extraction at the immune synapse. Mol. Biol. Cell 26, 1273-1285 (2015).

18. Yi, J. et al. Centrosome repositioning in $\mathrm{T}$ cells is biphasic and driven by microtubule end-on capture-shrinkage. J. Cell Biol. 202, 779-792 (2013).

19. Combs, J. et al. Recruitment of dynein to the Jurkat immunological synapse. Proc. Natl Acad. Sci. USA. 103, 14883-14888 (2006).

20. Martin-Cofreces, N. B. et al. MTOC translocation modulates IS formation and controls sustained T cell signaling. J. Cell Biol. 182, 951-962 (2008).

21. Gomez, T. S. et al. Formins regulate the actin-related protein $2 / 3$ complex independent polarization of the centrosome to the immunological synapse. Immunity 26, 177-190 (2007).

22. Schnyder, T. et al. B cell receptor-mediated antigen gathering requires ubiquitin ligase $\mathrm{Cbl}$ and adaptors Grb2 and Dok-3 to recruit dynein to the signaling microcluster. Immunity 34, 905-918 (2011).

23. Hoedt, E., Zhang, G. \& Neubert, T. A. Stable isotope labeling by amino acids in cell culture (SILAC) for quantitative proteomics. Adv. Exp. Med. Biol. 806, 93-106 (2014).

24. Rotty, J. D., Wu, C. \& Bear, J. E. New insights into the regulation and cellular functions of the ARP2/3 complex. Nat. Rev. Mol. Cell Biol. 14, 7-12 (2013).

25. Hubert, T., Vandekerckhove, J. \& Gettemans, J. Actin and Arp2/3 localize at the centrosome of interphase cells. Biochem. Biophys. Res. Commun. 404, 153-158 (2011).

26. Farina, F. et al. The centrosome is an actin-organizing centre. Nat. Cell Biol. 18, 65-75 (2016).

27. Nolen, B. J. et al. Characterization of two classes of small molecule inhibitors of Arp2/3 complex. Nature 460, 1031-1034 (2009).

28. Burke, T. A. et al. Homeostatic actin cytoskeleton networks are regulated by assembly factor competition for monomers. Curr. Biol. 24, 579-585 (2014).

29. Gomez, T. S. et al. HS1 functions as an essential actin-regulatory adaptor protein at the immune synapse. Immunity 24, 741-752 (2006).

30. Hao, J. J., Carey, G. B. \& Zhan, X. Syk-mediated tyrosine phosphorylation is required for the association of hematopoietic lineage cell-specific protein 1 with lipid rafts and B cell antigen receptor signalosome complex. J. Biol. Chem. 279, 33413-33420 (2004)

31. Monfregola, J., Napolitano, G., D’Urso, M., Lappalainen, P. \& Ursini, M. V. Functional characterization of Wiskott-Aldrich syndrome protein and scar homolog (WASH), a bi-modular nucleation-promoting factor able to interact with biogenesis of lysosome-related organelle subunit 2 (BLOS2) and gamma-tubulin. J. Biol. Chem. 285, 16951-16957 (2010).

32. Luxton, G. W., Gomes, E. R., Folker, E. S., Vintinner, E. \& Gundersen, G. G. Linear arrays of nuclear envelope proteins harness retrograde actin flow for nuclear movement. Science 329, 956-959 (2010).
33. Burakov, A. V. \& Nadezhdina, E. S. Association of nucleus and centrosome: magnet or velcro? Cell Biol. Int. 37, 95-104 (2013).

34. Maro, B. \& Bornens, M. The centriole-nucleus association: effects of cytochalasin B and nocodazole. Biol. Cell. 39, 287-290 (1980).

35. Karsenti, E., Kobayashi, S., Mitchison, T. \& Kirschner, M. Role of the centrosome in organizing the interphase microtubule array: properties of cytoplasts containing or lacking centrosomes. J. Cell Biol. 98, 1763-1776 (1984).

36. Kwon, M., Bagonis, M., Danuser, G. \& Pellman, D. Direct microtubule-binding by myosin-10 orients centrosomes toward retraction fibers and subcortical actin clouds. Dev. Cell 34, 323-337 (2015).

37. Kim, D. H., Cho, S. \& Wirtz, D. Tight coupling between nucleus and cell migration through the perinuclear actin cap. J. Cell Sci. 127, 2528-2541 (2014).

38. Kutscheidt, S. et al. FHOD1 interaction with nesprin-2G mediates TAN line formation and nuclear movement. Nat. Cell Biol. 16, 708-715 (2014).

39. Higgs, H. N., Blanchoin, L. \& Pollard, T. D. Influence of the C terminus of Wiskott-Aldrich syndrome protein (WASp) and the Arp2/3 complex on actin polymerization. Biochemistry 38, 15212-15222 (1999).

40. Sims, T. N. et al. Opposing effects of PKCtheta and WASp on symmetry breaking and relocation of the immunological synapse. Cell 129, 773-785 (2007).

41. Finetti, F. et al. Intraflagellar transport is required for polarized recycling of the TCR/CD3 complex to the immune synapse. Nat. Cell Biol. 11, 1332-1339 (2009).

42. de la Roche, M. et al. Hedgehog signaling controls $\mathrm{T}$ cell killing at the immunological synapse. Science 342, 1247-1250 (2013).

43. Malherbe, L. et al. Selective activation and expansion of high-affinity CD4+ $\mathrm{T}$ cells in resistant mice upon infection with Leishmania major. Immunity 13, 771-782 (2000).

44. Derivery, E. et al. The Arp2/3 activator WASH controls the fission of endosomes through a large multiprotein complex. Dev. Cell 17, 712-723 (2009).

45. Gogendeau, D., Guichard, P. \& Tassin, A. M. Purification of centrosomes from mammalian cell lines. Methods Cell Biol. 129, 171-189 (2015).

46. Burgo, A. et al. Increased activity of the vesicular soluble N-ethylmaleimidesensitive factor attachment protein receptor TI-VAMP/VAMP7 by tyrosine phosphorylation in the Longin domain. J. Biol. Chem. 288, 11960-11972 (2013).

47. Poullet, P., Carpentier, S. \& Barillot, E. myProMS, a web server for management and validation of mass spectrometry-based proteomic data. Proteomics 7, 2553-2556 (2007).

48. Yang, Y. H. et al. Normalization for cDNA microarray data: a robust composite method addressing single and multiple slide systematic variation. Nucleic Acids Res. 30, e15 (2002).

49. Boyle, E. I. et al. GO::TermFinder-open source software for accessing Gene Ontology information and finding significantly enriched Gene Ontology terms associated with a list of genes. Bioinformatics 20, 3710-3715 (2004).

50. MacLean-Fletcher, S. \& Pollard, T. D. Identification of a factor in conventional muscle actin preparations which inhibits actin filament self-association. Biochem. Biophys. Res. Commun. 96, 18-27 (1980).

51. Isambert, H. et al. Flexibility of actin filaments derived from thermal fluctuations. Effect of bound nucleotide, phalloidin, and muscle regulatory proteins. J. Biol. Chem. 270, 11437-11444 (1995).

52. Egile, C. et al. Activation of the CDC42 effector N-WASP by the Shigella flexneri IcsA protein promotes actin nucleation by Arp2/3 complex and bacterial actin-based motility. J. Cell Biol. 146, 1319-1332 (1999).

53. Fedorov, A. A., Pollard, T. D. \& Almo, S. C. Purification, characterization and crystallization of human platelet profilin expressed in Escherichia coli. J. Mol. Biol. 241, 480-482 (1994).

54. Schindelin, J. et al. Fiji: an open-source platform for biological-image analysis. Nat. Methods 9, 676-682 (2012).

\section{Acknowledgements}

We acknowledge P. Bousso, M. Bornens, P. Vargas, M. Chabaud, M. Bretou, V. Randrian and H. D. Moreau for critical discussions; M. Piel and C. Hivroz for critical reading of the manuscript; R. Basto and D. Gogendeau for advice on centrosome manipulation; G. Arras for his help with GO term enrichment analysis; and D. Lankar for technical advice and discussions. We thank Edgar R. Gomes for providing the Nesprin-2 SRKASH (LINC-DN) plasmid. We acknowledge the Nikon Imaging Center@CNRS-Institut Curie and PICT-IBiSA, Institut Curie, Paris, member of the France-BioImaging national research infrastructure, for support in image acquisition. D.O. was supported by fellowships from the Ecole Doctorale BioSPC, Université Paris Diderot and Université Paris Descartes and the Fondation pour la Recherche Médicale. M.I.-Y. was supported by a research grant from FONDECYT No. 1141182. Funding was obtained from the Association Nationale pour la Recherche (ANR-PoLyBex-12-BSV3-0014-001 to A.-M.L.-D. and ANR-12-BSV5-0004-01 to M.T. and L.B.) and the European Research Council (ERC-Strapacemi-GA 243103 to A.-M.L.-D. and Starting grant 310472 to M.T.).

\section{Author contributions}

D.O. designed, performed and analysed most of the experiments, assembled figures and participated in manuscript writing; F.F. and J.G. performed and analysed in vitro nucleation assays; O.M. assisted in cell culture, immunofluorescences, centrosome 
purifications and performed biochemistry experiments; P.J.S. performed calcium measurements and time-lapse experiments; M.M. wrote Fiji (ImageJ) routines for image analysis; F.D. carried out the LC-MS/MS experimental work and D.L. supervised LC-MS/MS and proteomic data analysis; A.G. produced and provided the antibody against WASH; M.-I.Y. adapted the protocol for, assisted in centrosome purifications and proposed the original hypothesis of changes in the centrosome proteome triggered by BCR engagement; L.B. and M.T. designed and supervised in vitro nucleation assays and participated in manuscript writing; A.-M.L.-D. designed and supervised the overall research, funded it and wrote the manuscript.

\section{Additional information}

Supplementary Information accompanies this paper at http://www.nature.com/ naturecommunications

Competing financial interests: The authors declare no competing financial interests.

Reprints and permission information is available online at http://npg.nature.com/ reprintsandpermissions/

How to cite this article: Obino, D. et al. Actin nucleation at the centrosome controls lymphocyte polarity. Nat. Commun. 7:10969 doi: 10.1038/ncomms10969 (2016).

(c) (i) article are included in the article's Creative Commons license, unless indicated otherwise in the credit line; if the material is not included under the Creative Commons license, users will need to obtain permission from the license holder to reproduce the material. To view a copy of this license, visit http://creativecommons.org/licenses/by/4.0/ 